Universalidade e ortogonalidade em espaços de Hilbert de reprodução

\author{
Victor Simões Barbosa
}





\title{
Universalidade e ortogonalidade em espaços de Hilbert de reprodução
}

\author{
Victor Simões Barbosa ${ }^{1}$
}

Orientador: Prof. Dr. Valdir Antonio Menegatto

Dissertação apresentada ao Instituto de Ciências Matemáticas e de Computação - ICMC-USP, como parte dos requisitos para obtenção do título de Mestre em Ciências - Matemática . VERSÃO REVISADA

USP - São Carlos

Abril de 2013

${ }^{1} \mathrm{O}$ autor teve apoio financeiro da FAPESP, Processo no 2010/13025-3. 
Ficha catalográfica elaborada pela Biblioteca Prof. Achille Bassi e Seção Técnica de Informática, ICMC/USP, com os dados fornecidos pelo(a) autor(a)

\begin{tabular}{|c|c|}
\hline \multirow[t]{3}{*}{ B228u } & $\begin{array}{l}\text { Barbosa, Victor } \\
\text { Universalidade e ortogonalidade em espaços de } \\
\text { Hilbert de reprodução / Victor Barbosa; orientador } \\
\text { Valdir Antonio Menegatto. -- São Carlos, } 2013 . \\
\quad 68 \text { p. }\end{array}$ \\
\hline & $\begin{array}{l}\text { Dissertação (Mestrado - Programa de Pós-Graduação } \\
\text { em Matemática) -- Instituto de Ciências Matemáticas } \\
\text { e de Computação, Universidade de São Paulo, } 2013 \text {. }\end{array}$ \\
\hline & $\begin{array}{l}\text { 1. Núcleos positivos definidos. 2. Função layout. } \\
\text { 3. Espaços de Hilbert de reprodução. } 4 \text {. } \\
\text { Universalidade. 5. Ortogonalidade. I. Menegatto, } \\
\text { Valdir Antonio, orient. II. Título. }\end{array}$ \\
\hline
\end{tabular}




\section{Agradecimentos}

Agradeço primeiramente a Deus, pois sem Ele todo este trabalho ainda estaria por ser feito.

Aos meus pais Hamilton e Gisele por serem as pessoas mais fantásticas que conheço e por nunca medirem esforços para possibilitar que eu atingisse meus objetivos.

À minha irmã Amanda e ao meu irmão José Luiz por completarem esta família maravilhosa a qual devo muito do que sou e tenho hoje.

Aos meus quase irmãos e grandes amigos Francys e Lucas pela companhia e todo o apoio que foram fundamentais para superar as dificuldades.

À minha namorada Patricia pela imensa paciência e compreensão, pelo enorme carinho e cuidado durante todo o período de confecção do presente trabalho e por centenas de outros motivos que contribuiram de forma imprescindivel para que eu chegasse até aqui.

À Prof ${ }^{a}$. Dra. Marta Cilene Gadotti pela ajuda de sempre.

Aos amigos do ICMC: Alina, Ariadne, Evandro, Gabriel, Gil, Laís, Noemi, Preta, Thaís, dentre tantos outros com quem tive o prazer de dividir inúmeros momentos dando-me a certeza que nunca estive só nesta caminhada.

Aos funcionários do ICMC, em especial às secretárias Ana Carolina, Ana Paula, Glaucia e Lhaís por toda a atenção e competência.

Expresso ainda minha sincera gratidão ao meu orientador Prof. Dr. Valdir Antonio Menegatto pela dedicação, paciência, amizade e principalmente por toda a confiança depositada sobre mim.

Por fim agradeço a FAPESP pelo apoio financeiro. 

Neste trabalho analisamos o papel das funções layout de um núcleo positivo definido $K$ sobre um espaço topológico de Hausdorff $E$ com relação a duas propriedades específicas: a universalidade de $K$ e a ortogonalidade no espaço de Hilbert de reprodução de $K$ a partir de suportes disjuntos. As funções layout sempre existem mas podem não ser únicas. De uma maneira geral, a função layout é uma aplicação que transfere, convenientemente, informações do espaço $E$ para um espaço com produto interno de dimensão alta, onde métodos lineares podem ser usados. Tanto a universalidade quanto a ortogonalidade pressupõem a continuidade do núcleo. O primeiro conceito exige que para cada compacto não vazio $X$ de $E$, o conjunto de "seções" $\{K(\cdot, y): y \in X\}$ seja total no espaço de todas as funções contínuas com domínio $X$, munido da topologia da convergência uniforme. Um dos resultados principais do trabalho caracteriza a universalidade de um núcleo $K$ através de uma propriedade de universalidade semelhante da função layout. A ortogonalidade a partir de suportes disjuntos almeja, então, a ortogonalidade de quaisquer duas funções do espaço de Hilbert de reprodução de $K$ quando seus suportes não se intersectam. 

We analyze the role of feature maps of a positive definite kernel $K$ acting on a Hausdorff topological space $E$ in two specific properties: the universality of $K$ and the orthogonality in the reproducing kernel Hilbert space of $K$ from disjoint supports. Feature maps always exist but may not be unique. A feature map may be interpreted as a kernel based procedure that maps the data from the original input space $E$ into a potentially higher dimensional "feature space" in which linear methods may then be used. Both properties, universality and orthogonality from disjoint supports, make sense under continuity of the kernel. Universality of $K$ is equivalent to the fundamentality of $\{K(\cdot, y): y \in X\}$ in the space of all continuous functions on $X$, with the topology of uniform convergence, for all nonempty compact subsets $X$ of $E$. One of the main results in this work is a characterization of the universality of $K$ from a similar concept for the feature map. Orthogonality from disjoint supports seeks the orthogonality of any two functions in the reproducing kernel Hilbert space of $K$ when the functions have disjoint supports. 



\section{Sumário}

\begin{tabular}{ll}
\hline Introdução & 13
\end{tabular}

\begin{tabular}{lll}
\hline & Preliminares & 15
\end{tabular}

1.1 Núcleos positivos definidos e a função layout . . . . . . . . . . . . . . . 15

1.2 Existência de funções layout para um núcleo positivo definido . . . . . 17

1.3 Funções layout e espaços de Hilbert de reprodução . . . . . . . . . . . . . 22

1.4 Espaços de Hilbert de Reprodução de dimensão finita . . . . . . . . . . . 25

$\begin{array}{lll}2 & \text { Núcleos universais } & 29\end{array}$

2.1 O teorema de Riesz e a função layout . . . . . . . . . . . . . . . . . . . 29

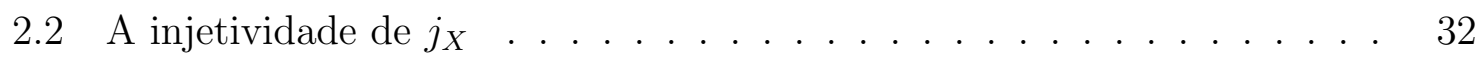

2.3 A aplicação $l_{X}$ e o seu papel . . . . . . . . . . . . . . . . . . . . 35

2.4 Universalidade . . . . . . . . . . . . . . . . . . . . . . . . . . . . . . . . . 37

2.5 Universalidade e a teoria de Mercer . . . . . . . . . . . . . . . . . . . . . 38

\begin{tabular}{|lll}
\hline 3 & Exemplificando universalidade & 43
\end{tabular}

3.1 Um núcleo de Mercer clássico . . . . . . . . . . . . . . . . . . . . . . . 43

3.2 Núcleos definidos por séries de potências . . . . . . . . . . . . . . . . . 44

3.3 Núcleos PD sobre a esfera unitária $\ldots \ldots \ldots$. . . . . . . . . . . . . . . . 46

3.4 Núcleos invariantes por translação sobre $\mathbb{R}^{d} \ldots \ldots$. . . . . . . . . . 47

3.5 Núcleos radiais sobre $\mathbb{R}^{d} \ldots \ldots \ldots \ldots \ldots$. . . . . . . . . . . . . 52

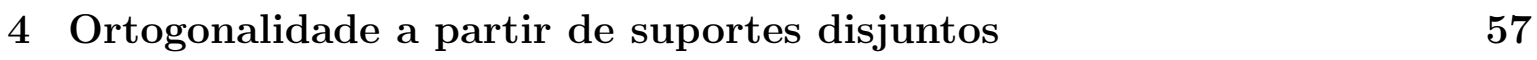

4.1 Conceitos preliminares e um exemplo . . . . . . . . . . . . . . . 57

4.2 Ortogonalidade via suportes disjuntos e a função layout . . . . . . . . . 58

4.3 Exemplificando . . . . . . . . . . . . . . . . . . . . . . . . . 60 
\begin{tabular}{ll}
\hline Referências Bibliográficas & 63
\end{tabular}

$\begin{array}{ll}\text { Índice Remissivo } & 67\end{array}$ 
Os núcleos positivos definidos aparecem em vários segmentos da Matemática, incluindo Álgebra Linear, Análise Funcional, Teoria da Aproximação, etc.([8, 25]). Mais recentemente, eles têm sido usados com relativa frequência na Teoria do Aprendizado ([9]), em problemas envolvendo a análise espectral fina de operadores integrais ([3, 10]) bem como em várias outras instâncias em que espaços de Hilbert de reprodução e espaços nativos aparecem nas formulações dos problemas ([4, 17, 25]).

A teoria dos núcleos positivos definidos é composta por uma gama considerável de objetos, dentre os quais podemos destacar as chamadas funções layout (feature maps) atreladas a eles. Estas funções têm papel fundamental nessa teoria já que elas transferem, via o núcleo, propriedades do conjunto domínio do núcleo para um espaço com produto interno conveniente. O objetivo principal deste trabalho é discutir um pouco da teoria dos núcleos positivos definidos sobre um espaço topológico, focando naquelas propriedades que envolvem diretamente as funções layout. Assim sendo, tais funções tem papel determinante nos principais resultados abordados nesta dissertação.

Inicialmente fazemos uma breve introdução da teoria dos núcleos positivos definidos necessária para o entendimento do trabalho. Entre definições, exemplos e resultados, exploramos diversas propriedades: a existência e unicidade do espaço de Hilbert de reprodução associado ao núcleo, a representação do núcleo via função layout e a descrição do espaço de Hilbert de reprodução via função layout. Neste último ponto, analisamos a fundo a existência de tal representação e discutimos a sua não unicidade. Ademais, apresentamos duas formulações diferentes para explicitar o espaço de Hilbert de reprodução em termos de uma função layout, dependendo da presença ou ausência de uma propriedade de fundamentalidade.

Posteriormente, trabalhando tão somente com núcleos positivos definidos contínuos, definimos formalmente o que significa o núcleo possuir a propriedade universal da aproximação (ou resumidamente ser um núcleo universal). Partindo desta definição, 
buscamos condições para garantir a universalidade do núcleo, de maneira paralela ao estudo desenvolvido em [16]. Em um dos principais resultados do trabalho, encontramos uma condição necessaria e suficiente sobre a função layout para que um núcleo seja universal. Frente as dificuldades existentes, vimo-nos na necessidade de definir diversos objetos, buscando formas de relacioná-los, até obtermos o resultado desejado. Estas definições e relações exigiram conceitos nem sempre tão básicos de integração, teoria da medida, análise funcional clássica, entre outros.

Na sequência, fixando o núcleo dentro de alguma categoria definida, exploramos diferentes condições sobre os mesmos para garantir a propriedade universal da aproximação. Para um núcleo de Mercer clássico, apresentamos uma condição para universalidade envolvendo as aplicações que compõem sua representação. Amparados em resultados envolvendo multi-índices, justificamos a universalidade de núcleos definidos por séries de potências em várias variáveis reais. Análises similares foram implementadas para determinar a universalidade de núcleos positivos definidos na esfera unitária $m$ dimensional, de núcleos radiais sobre $\mathbb{R}^{n}$ e de núcleos sobre $\mathbb{R}^{n}$ invariantes por translações.

Por último, examinamos os espaços de Hilbert de reprodução associados a núcleos positivos definidos contínuos, tentando identificar hipóteses adicionais para garantir a ortogonalidade a partir de suportes disjuntos no espaço. Tal propriedade reflete a ortogonalidade de quaisquer duas funções do espaço, quando as mesmas têm suportes disjuntos. Nossa análise baseou-se na referência [27] e tentou usar, tanto quanto possível, as funções layout previamente estudadas. 


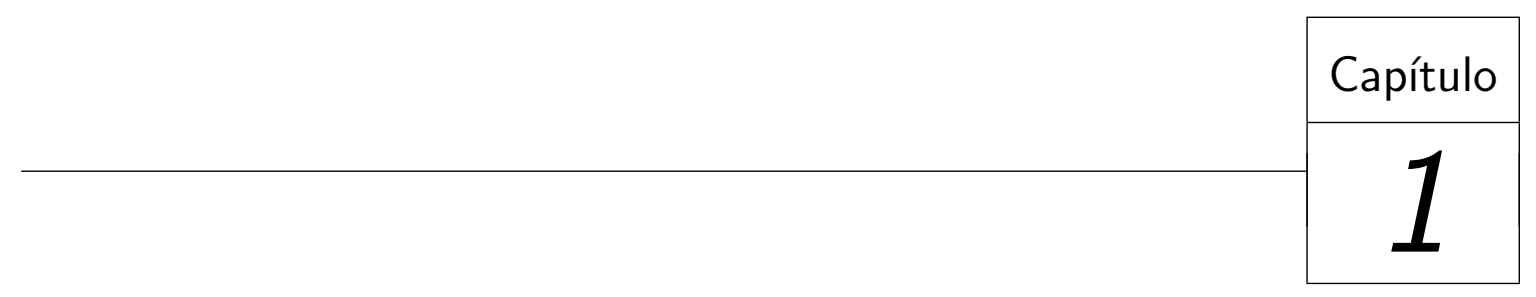

Preliminares

Neste capítulo, introduziremos os conceitos necessários ao desenvolvimento do trabalho, exemplificando e apresentando os resultados básicos atrelados a eles. Isso será feito de maneira econômica, ou seja, não exploraremos estes conceitos além daquilo que o trabalho exigirá. Referências que contém informações adicionais ou mesmo resultados mais profundos envolvendo estes conceitos serão indicadas ao longo do texto.

\subsection{Núcleos positivos definidos e a função layout}

A seguinte definição contempla a definição usual de núcleo positivo definido. Aqui $E$ é um conjunto não vazio qualquer.

Definição 1.1.1. Uma função $K: E \times E \rightarrow \mathbb{C}$ é um núcleo positivo definido sobre $E$ (resumidamente, núcleo $P D$ sobre $E$ ) se a matriz $n \times n$ com entradas $K\left(x_{i}, x_{j}\right)$ é não negativa definida para todo inteiro positivo $n$ e quaisquer $n$ pontos $x_{1}, x_{2}, \ldots, x_{n}$ de $E$.

Em outras palavras, a definição acima exige que

$$
\sum_{j, k=1}^{n} c_{j} \overline{c_{k}} K\left(x_{j}, x_{k}\right) \geq 0
$$

para todo inteiro positivo $n$ e quaisquer que sejam os pontos $x_{1}, x_{2}, \ldots, x_{n}$ de $E$ e os números complexos $c_{1}, c_{2}, \ldots, c_{n}$.

Núcleos PD genuinamente complexos são automaticamente hermitianos. Se o núcleo é uma função a valores reais, e esta mesma propriedade é desejável, então ela tem que 
ser adicionada ao contexto. Uma teoria para tais núcleos pode ser encontrada em [3]. A referência [21] traz núcleos positivos definidos em algoritmos para análise de padrão (pattern analysis) e na teoria do aprendizado (learning theory), temas mais modernos onde os mesmos se apresentam.

Agora, ilustraremos a definição acima com alguns poucos exemplos.

Exemplo 1.1.2. Se $E$ é um espaço vetorial complexo com produto interno $\langle\cdot, \cdot\rangle_{E}$, então a expressão

$$
K(x, y)=\langle x, y\rangle_{E}, \quad x, y \in E,
$$

define um núcleo PD sobre $E$. De fato, basta notar que

$$
\sum_{i, j=1}^{n} c_{i} \overline{c_{j}} K\left(x_{i}, x_{j}\right)=\sum_{i, j=1}^{n} c_{i} \overline{c_{j}}\left\langle x_{i}, x_{j}\right\rangle_{E}=\left\langle\sum_{i=1}^{n} c_{i} x_{i}, \sum_{j=1}^{n} c_{j} x_{j}\right\rangle_{E} \geq 0
$$

quaisquer que sejam os números complexos $c_{j}$ 's e os elementos $x_{j}$ 's de $E$.

Exemplo 1.1.3. Se $f$ é uma função complexa com domínio $E$, então a expressão

$$
K(x, y)=f(x) \overline{f(y)}, \quad x, y \in X
$$

define um núcleo PD sobre $E$.

Exemplo 1.1.4. Seja $d$ um inteiro positivo fixado e considere a esfera unitária $S^{d}$ de $\mathbb{R}^{d+1}$. Seja $P_{k}^{d}, k \in \mathbb{Z}_{+}$, o polinômio de Legendre de grau $k$ associado ao inteiro positivo $d$, como discutido em [12]. Este polinômio é um múltiplo do polinômio ultra-esférico $P_{k}^{(d-1) / 2}$ de grau $k$ associado ao número real $(d-1) / 2$ como descrito em [22] e carrega a normalização $P_{k}^{d}(1)=1$. A expressão

$$
K(x, y)=\sum_{k=0}^{\infty} a_{k} P_{k}^{d}(x \cdot y), \quad x, y \in \mathbb{S}^{d},
$$

onde "." é o produto interno usual de $\mathbb{R}^{d+1}$, cada $a_{k}$ é não negativo e $\sum_{k=0}^{\infty} a_{k}<\infty$, define um núcleo PD contínuo sobre $S^{d}$. Este resultado é uma parte da caracterização dos núcleos PD contínuos e bizonais fornecida por Schoenberg em [20]. Em particular, cada função $(x, y) \in S^{d} \times S^{d} \rightarrow P_{k}^{d}(x \cdot y)$ é um núcleo PD sobre $S^{d}$.

A proposição abaixo descreve uma generalização dos dois primeiros exemplos citados acima.

Proposição 1.1.5. Seja $\mathcal{W}$ um espaço com produto interno $\langle\cdot, \cdot\rangle_{\mathcal{W}}$. Se uma aplicação $K: E \times E \rightarrow \mathbb{C}$ satisfaz

$$
K(x, y)=\langle\Phi(x), \Phi(y)\rangle_{\mathcal{W}}, \quad x, y \in E
$$

para alguma função $\Phi: E \rightarrow \mathcal{W}$, então $K$ é um núcleo PD sobre $E$. 
Demonstração: Se $K$ é como na fórmula do enunciado da proposição, a forma quadrática da Definição 1.1.1 é

$$
\sum_{j, k=1}^{n} c_{j} \overline{c_{k}}\left\langle\Phi\left(x_{j}\right), \Phi\left(x_{k}\right)\right\rangle=\left\langle\sum_{j=1}^{n} c_{j} \Phi\left(x_{j}\right), \sum_{k=1}^{n} c_{k} \Phi\left(x_{k}\right)\right\rangle_{\mathcal{W}},
$$

uma expressão sempre não negativa.

A proposição acima motiva a seguinte definição.

Definição 1.1.6. Seja K um núcleo PD sobre $E$. Se existir um espaço de Hilbert $\mathcal{W}$ munido do produto interno $\langle\cdot, \cdot\rangle_{\mathcal{W}}$ e uma aplicação $\Phi: E \rightarrow \mathcal{W}$ tal que

$$
K(x, y)=\langle\Phi(x), \Phi(y)\rangle_{\mathcal{W}}, \quad x, y \in E
$$

diremos que $\Phi$ é uma função layout para o núcleo $K$.

Usualmente, o espaço $\mathcal{W}$ da definição acima é chamado de um espaço layout para $K$, mas esta terminologia não será explorada nesta dissertação. Como a função $\Phi$ pode não ser sobrejetora e o espaço $\mathcal{W}$ é passível de restrições ou extensões métricas, é de se esperar que quando uma função layout para $K$ existe, ela não é necessariamente única. Além disso, note que a condição de $\mathcal{W}$ ser um espaço de Hilbert não é necessária para garantir que o núcleo $K$ seja positivo definido, porém a construção que será feita adiante nos fornece a garantia de que tal condição pode ser incorporada à Definição 1.1.6, sem perda alguma de generalidade. A existência e construção de funções layout para um núcleo positivo definido $K$ é o tema da seção seguinte.

\subsection{Existência de funções layout para um núcleo posi- tivo definido}

Apresentaremos nesta seção dois métodos consideravelmente diferentes para se construir funções layout para um núcleo PD sobre um conjunto não vazio. Em particular, isto ratificará a não unicidade da função layout mencionada no final da seção anterior.

Começaremos recordando a definição de completamento para um espaço com produto interno.

Definição 1.2.1. Seja E um espaço com produto interno $\langle\cdot, \cdot\rangle_{E}$. Diremos que um espaço de Hilbert $\left(\tilde{E},\langle\cdot, \cdot\rangle_{\tilde{E}}\right)$ é o completamento de $\left(E,\langle\cdot, \cdot\rangle_{E}\right)$, se existir uma aplicação $T: E \rightarrow \tilde{E}$ linear e injetora satisfazendo

(i) $\langle T(x), T(y)\rangle_{\tilde{E}}=\langle x, y\rangle_{E}$;

(ii) $T(E)$ é denso em $\tilde{E}$. 
Enunciaremos agora um importante resultado sobre a existência do completamento de um espaço com produto interno. Sua demonstração pode ser encontrada em [15, p.139].

Lema 1.2.2. Todo espaço com produto interno $E$ admite um completamento $\tilde{E}$. Ademais, este completamento é único a menos de isomorfismos isométricos.

Dando sequência, definiremos formalmente espaço de Hilbert de reprodução (EHR). Se $K$ é um núcleo sobre um conjunto não vazio $E$ e $x \in E$, denotaremos por $K_{x}$ a função $y \in E \rightarrow K(x, y)$.

Definição 1.2.3. Seja $K$ um núcleo PD sobre um conjunto não vazio E. O espaço de Hilbert de reprodução associado a $K$ é um espaço de Hilbert $\left(\mathcal{H}_{K},\langle\cdot, \cdot\rangle\right)$ (de funções de $E$ em $\mathbb{C})$ que satisfaz as seguintes propriedades:

(i) $K_{x} \in \mathcal{H}_{K}, x \in E$;

(ii) (Propriedade de reprodução) $\left\langle f, K_{x}\right\rangle=f(x), f \in \mathcal{H}_{K}, x \in E$.

A teoria dos espaços de Hilbert de reprodução está bem fundamentada na literatura ([1, 19]). A existência e unicidade do EHR associado a um núcleo $K$ são justificadas abaixo. Antes, porém, lembremos que se $U$ é um subconjunto de um espaço de Hilbert $\mathcal{H}$, o complemento ortogonal $U^{\perp}$ de $U$ em $\mathcal{H}$ é o subespaço formado por todos os elementos de $\mathcal{H}$ que são ortogonais a cada elemento de $U$.

Teorema 1.2.4. Se K é um núcleo PD sobre um conjunto não vazio E, então existe um espaço de Hilbert de reprodução associado a $K$, o qual é único a menos de isomorfismos isométricos.

Demonstração: Consideremos o espaço de funções

$$
\mathcal{H}_{0}=\left[\left\{K_{y}: y \in E\right\}\right]
$$

Para duas funções quaisquer em $\mathcal{H}_{0}$, digamos

$$
f(\cdot)=\sum_{i=1}^{m} \alpha_{i} K\left(\cdot, x_{i}\right) \text { e } g(\cdot)=\sum_{j=1}^{n} \beta_{j} K\left(\cdot, y_{j}\right)
$$

a fórmula

$$
\langle f, g\rangle_{\mathcal{H}_{0}}:=\sum_{i=1}^{m} \sum_{j=1}^{n} \alpha_{i} \overline{\beta_{j}} K\left(y_{j}, x_{i}\right)
$$

define uma aplicação bilinear hermitiana de $\mathcal{H}_{0} \times \mathcal{H}_{0}$ em $\mathbb{C}$. Como $K$ é PD sobre $E$, segue que $\langle f, f\rangle_{\mathcal{H}_{0}} \geq 0, f \in \mathcal{H}_{0}$. Em particular, a fórmula $\|f\|_{\mathcal{H}_{0}}=\langle f, f\rangle_{\mathcal{H}_{0}}, f \in \mathcal{H}_{0}$, 
define uma semi-norma em $\mathcal{H}_{0}$. Agora, se $\langle f, f\rangle_{\mathcal{H}_{0}}=0$, podemos usar a desigualdade de Cauchy-Schwarz para deduzir que

$$
|f(x)|=\left|\langle f, K(\cdot, x)\rangle_{\mathcal{H}_{0}}\right| \leq\|f\|_{\mathcal{H}_{0}}\|K(\cdot, x)\|_{\mathcal{H}_{0}}=0, \quad x \in E .
$$

Em outras palavras, $f=0$. Desta forma, $\left(\mathcal{H}_{0},\langle\cdot, \cdot\rangle_{\mathcal{H}_{0}}\right)$ é um espaço com produto interno. Como

$$
\langle f, K(\cdot, x)\rangle_{\mathcal{H}_{0}}=\sum_{i=1}^{m} \alpha_{i} K\left(x, x_{i}\right)=f(x), \quad x \in E,
$$

as propriedades $(i)$ e $(i i)$ da definição de EHR já valem, mesmo $\left(\mathcal{H}_{0},\langle\cdot, \cdot\rangle_{\mathcal{H}_{0}}\right)$ não sendo ainda um espaço de Hilbert. O completamento $\left(\mathcal{H},\|\cdot\|_{\mathcal{H}}\right)$ de $\left(\mathcal{H}_{0},\|\cdot\|_{\mathcal{H}_{0}}\right)$, conforme descrito no início desta seção, é um EHR associado a $K$. Para mostramos que ele é único a menos de isomorfismos isométricos, suponhamos que $\left(\mathcal{H}_{1},\langle\cdot, \cdot\rangle_{\mathcal{H}_{1}}\right)$ seja outro EHR associado $K$. A definição de $\mathcal{H}_{0}$ implica imediatamente que $\mathcal{H}_{0} \subseteq \mathcal{H}_{1}$ e pela propriedade de reprodução válida em $\mathcal{H}_{1}$, segue que

$$
\langle f, g\rangle_{\mathcal{H}_{0}}=\langle f, g\rangle_{\mathcal{H}_{1}}, \quad f, g \in \mathcal{H}_{0}
$$

Consideremos a aplicação inclusão $\mathcal{H}_{0} \stackrel{i}{\longrightarrow} \mathcal{H}_{1}$. É de conhecimento prévio que $i$ é linear e injetora. Além disso (1.1) nos garante que $i$ satisfaz a condição $(i)$ na definição de completamento. Por fim, mostremos que $\overline{i\left(\mathcal{H}_{0}\right)}=\mathcal{H}_{1}$. Observemos que se esta igualdade não fosse verdadeira, então a inclusão $\overline{i\left(\mathcal{H}_{0}\right)} \subseteq \mathcal{H}_{1}$ seria própria, logo poderíamos selecionar uma função $g$ não nula no complemento ortogonal de $\overline{i\left(\mathcal{H}_{0}\right)}$ em $\mathcal{H}_{1}$. No entanto, a propriedade de reprodução em $\mathcal{H}_{1}$ implicaria que

$$
g(x)=\left\langle g, K_{x}\right\rangle_{\mathcal{H}_{1}}=0, \quad x \in E
$$

uma contradição. Assim, temos a igualdade sugerida e pelo Lema 1.2.2, concluímos o resultado.

A existência de um $\operatorname{EHR}\left(\mathcal{H}_{K},\langle\cdot, \cdot\rangle_{\mathcal{H}_{K}}\right)$ associado a um núcleo positivo definido $K$ sobre $E$ permite a construção imediata de uma função layout para $K$. De fato, como a construção do EHR apresentada na prova do teorema anterior nos dá

$$
K(x, y)=\left\langle K_{x}, K_{y}\right\rangle_{\mathcal{H}_{K}}, \quad x, y \in E
$$

basta definirmos $\mathcal{W}:=\mathcal{H}_{K}$ e $\Phi(x)=K_{x}, x \in E$.

Neste momento é importante observarmos que a construção apresentada no teorema anterior não aproveita qualquer estrutura adicional que o conjunto $E$ possa ter. A segunda construção que apresentaremos inclui algumas vantagens em relação à anterior, já que o espaço de Hilbert $\mathcal{W}$ resultante na definição de função layout é automaticamente separável, uma consequência do refinamento das hipóteses sobre $K$. 
Teorema 1.2.5. Seja E um conjunto não vazio e $K$ um núcleo PD sobre E. Suponha que $K$ admita uma representação na forma

$$
K(x, y)=\sum_{j=0}^{\infty} \lambda_{j} \phi_{j}(x) \overline{\phi_{j}(y)}, \quad(x, y) \in E \times E,
$$

onde cada $\lambda_{j}$ é positivo, cada $\phi_{j}$ é uma função com domínio $E$ a valores complexos e a série converge para cada par $(x, y)$ de $E \times E$. Então, existe uma função layout $\Phi: E \rightarrow \mathcal{W}$ para $K$, onde o espaço de Hilbert $\mathcal{W}$ é separável.

Demonstração: É suficiente tomarmos $\mathcal{W}$ como sendo o espaço de Hilbert $\ell_{2}$ usual e definirmos $\Phi: E \rightarrow \ell_{2}$ pela fórmula $\Phi(x)=(x(j)), x \in E$, onde

$$
x(j):=\lambda_{j}^{1 / 2} \phi_{j}(x), \quad x \in E, \quad j=0,1, \ldots
$$

De fato, denotando o produto interno de $\ell_{2}$ por $\langle\cdot, \cdot\rangle_{2}$, temos que

$$
\langle\Phi(x), \Phi(y)\rangle_{2}=\sum_{j=1}^{\infty} x(j) \overline{y(j)}=\sum_{j=1}^{\infty} \lambda_{j}^{1 / 2} \phi_{j}(x) \overline{\lambda_{j}^{1 / 2} \phi_{j}(y)}=K(x, y), \quad x, y \in E .
$$

Isto finaliza a demonstração do teorema.

A representação para $K$ usada como hipótese no teorema anterior está em consonância com a representação para núcleos fornecida pelo teorema de Mercer. O teorema vale em vários contextos, com hipóteses adequadas a cada um deles. A situação mais comum ocorre quando $E$ tem alguma topologia e $K$ é no mínimo contínuo. Por outro lado, o teorema de Mercer usualmente garante a convergência uniforme e absoluta da série que representa $K$ e a sequência de funções na representação é usualmente formada por funções contínuas. Indicamos a referência [10] e outras lá citadas para informações gerais sobre o teorema de Mercer e generalizações.

O teorema anterior é perfeitamente adaptável para o caso em que a série representando $K$ é uma soma finita. Uma formalização deste fato forma o conteúdo dos dois resultados a seguir. Usaremos $u^{*}$ para denotar o vetor obtido de $u \in \mathbb{C}^{d}$ via conjugação seguida por uma transposição.

Teorema 1.2.6. Seja E um conjunto não vazio e $K$ um núcleo PD sobre E. Então, $\mathcal{H}_{K}$ tem dimensão finita $d$ se, e somente se, existem uma matriz $A$ (que depende de $K)$ hermitiana e positiva definida de ordem d e um conjunto linearmente independente $\left\{\phi_{1}, \phi_{2}, \ldots, \phi_{d}\right\}$ de funções complexas com domínio $E$ de modo que

$$
K(x, y)=\phi(x) A \phi(y)^{*}, \quad x, y \in E
$$

onde $\phi(x)=\left(\phi_{1}(x), \phi_{2}(x), \ldots, \phi_{d}(x)\right), x \in E$. 
Demonstração: Suponha que $\mathcal{H}_{K}$ tenha dimensão $d$ e fixe uma base ortogonal $B=$ $\left\{\phi_{1}, \phi_{2}, \ldots, \phi_{d}\right\}$ de $\mathcal{H}_{K}$. Como $K_{x} \in \mathcal{H}_{K}, x \in E$, podemos escrever

$$
K_{x}=\sum_{j=1}^{d} \alpha_{i}^{x} \phi_{i}, \quad \alpha_{1}^{x}, \alpha_{2}^{x}, \ldots, \alpha_{d}^{x} \in \mathbb{C}, \quad x \in E .
$$

Por outro lado, a ortogonalidade de $B$ nos dá, para cada $x \in E$, que

$$
\left\langle K_{x}, \phi_{j}\right\rangle_{\mathcal{H}_{K}}=\left\langle\sum_{i=1}^{d} \alpha_{i}^{x} \phi_{i}, \phi_{j}\right\rangle_{\mathcal{H}_{K}}=\sum_{i=1}^{d} \alpha_{i}^{x}\left\langle\phi_{i}, \phi_{j}\right\rangle_{\mathcal{H}_{K}}=\alpha_{j}^{x}\left\|\phi_{j}\right\|_{\mathcal{H}_{K}}^{2}, \quad j=1,2, \ldots, d .
$$

Como cada $\phi_{j}$ não é identicamente nula, obtemos

$$
K_{x}=\sum_{j=1}^{d} \frac{\left\langle K_{x}, \phi_{j}\right\rangle_{\mathcal{H}_{K}}}{\left\|\phi_{j}\right\|_{\mathcal{H}_{K}}^{2}} \phi_{j}, \quad x \in E
$$

Pela propriedade de reprodução em $\mathcal{H}_{K}$, podemos agora deduzir que

$$
K(x, y)=\left\langle K_{x}, K_{y}\right\rangle_{\mathcal{H}_{K}}=\sum_{i, j=1}^{d} \frac{\phi_{i}(x)}{\left\|\phi_{i}\right\|_{\mathcal{H}_{K}}^{2}} \overline{\left(\frac{\phi_{j}(y)}{\left\|\phi_{j}\right\|_{\mathcal{H}_{K}}^{2}}\right)}\left\langle\phi_{i}, \phi_{j}\right\rangle_{\mathcal{H}_{K}}, \quad x, y \in E
$$

Rearranjando,

$$
K(x, y)=\sum_{i, j=1}^{d} \frac{\left\langle\phi_{i}, \phi_{j}\right\rangle_{\mathcal{H}_{K}}}{\left\|\phi_{i}\right\|_{\mathcal{H}_{K}}^{2}\left\|\phi_{j}\right\|_{\mathcal{H}_{K}}^{2}} \phi_{i}(x) \overline{\phi_{j}(y)}, \quad x, y \in E
$$

Esta é a fórmula do enunciado com $A=\left(a_{i j}\right)$, onde

$$
a_{i j}=\frac{\left\langle\phi_{i}, \phi_{j}\right\rangle_{\mathcal{H}_{K}}}{\left\|\phi_{i}\right\|_{\mathcal{H}_{K}}^{2}\left\|\phi_{j}\right\|_{\mathcal{H}_{K}}^{2}}, \quad i, j=1,2, \ldots, d .
$$

A recíproca segue do Teorema 1.2 .4 e observando-se que cada $K_{x}$ pertence ao espaço de dimensão finita $\left[\left\{\phi_{1}, \phi_{2}, \ldots, \phi_{d}\right\}\right]$.

Note que na primeira implicação da demonstração acima podemos tomar, em particular, $B$ como sendo uma base ortonormal para $\mathcal{H}_{K}$. Neste caso, a matriz $A$ torna-se a matriz identidade de ordem $d$ enquanto que a representação para o núcleo $K$ toma a forma simplificada

$$
K(x, y)=\sum_{i=1}^{d} \phi_{i}(x) \overline{\phi_{i}(y)}, \quad x, y \in E .
$$

Como toda matriz positiva definida tem uma raíz quadrada, o resultado a seguir dispensa demonstração. 
Corolário 1.2.7. Nas condições do teorema anterior, se $\mathcal{W}=\mathbb{C}^{d}$ com seu produto interno usual $\langle\cdot, \cdot\rangle_{\mathbb{C}^{d}}$ e $B$ é uma raíz quadrada de $A$, então $\Phi: E \rightarrow \mathbb{C}^{d}$ dada por $\Phi(x)=\phi(x) B, x \in E$, é uma função layout para $K$.

Finalizaremos esta seção, apresentando um resultado independente que atrela a continuidade do núcleo à continuidade de qualquer função layout para ele.

Proposição 1.2.8. Seja $K$ um núcleo PD sobre um espaço topológico E. Se $\Phi: E \rightarrow \mathcal{W}$ é uma função layout para $K$, então $K$ é contínuo se, e somente se, $\Phi$ é contínua.

Demonstração: Seja $\Phi: E \rightarrow \mathcal{W}$ é uma função layout para $K$ e denote a norma em $\mathcal{W}$ por $\|\cdot\|$. É fácil ver que

$$
\begin{aligned}
\|\Phi(x)-\Phi(y)\|^{2} & =\langle\Phi(x), \Phi(x)\rangle_{\mathcal{W}}+\langle\Phi(y), \Phi(y)\rangle_{\mathcal{W}}-\langle\Phi(x), \Phi(y)\rangle_{\mathcal{W}}-\langle\Phi(y), \Phi(x)\rangle_{\mathcal{W}} \\
& =K(x, x)+K(y, y)-K(x, y)-K(y, x) .
\end{aligned}
$$

Portanto, a continuidade de $K$ implica na continuidade de $\Phi$. Para a recíproca, basta usar a desigualdade

$|K(x, y)-K(z, w)| \leq\|\Phi(x)-\Phi(z)\|\|\Phi(y)\|+\|\Phi(z)\|\|\Phi(y)-\Phi(w)\|, \quad x, y, z, w \in E$.

Isto conclui a demonstração.

\subsection{Funções layout e espaços de Hilbert de reprodução}

Nesta seção, descreveremos dois resultados que estabelecem relações mais efetivas entre as funções layout e os espaços de Hilbert de reprodução.

Vejamos uma motivação para o primeiro deles. Seja $\Phi: E \rightarrow \mathcal{W}$ uma função layout para o núcleo $K \mathrm{PD}$ sobre $E$. Podemos extrair a seguinte fórmula da definição de função layout:

$$
K_{y}(x)=K(x, y)=\langle\Phi(x), \Phi(y)\rangle_{\mathcal{W}, \quad x, y \in E .}
$$

Se para $u \in \mathcal{W}$ fixo, colocarmos

$$
\Phi_{u}(x):=\langle\Phi(x), u\rangle_{\mathcal{W}}, \quad x \in E
$$

a fórmula anterior se torna

$$
K_{y}(x)=\Phi_{\Phi(y)}(x), \quad x \in E .
$$

Em particular, ela revela que

$$
\left\{K_{y}: y \in E\right\} \subseteq\left\{\Phi_{u}: u \in \mathcal{W}\right\},
$$


e, portanto, é razoável tentarmos descrever $\mathcal{H}_{K}$ através das funções $\Phi_{u}$.

Lembramos uma terminologia comum que usaremos ao longo do trabalho.

Definição 1.3.1. Um subconjunto não vazio $A$ de um espaço vetorial normado $V$ é fundamental em $V$ se o subespaço gerado por $A$ é denso em $V$. Dependendo do contexto, a palavra fundamental pode ser substituída pela palavra total.

Proposição 1.3.2. Sejam $K$ um núcleo $P D$ sobre $E$ e $\Phi: E \rightarrow \mathcal{W}$ uma função layout para $K$. Se $\Phi(E)$ é um subconjunto fundamental de $\mathcal{W}$, então $\left(\mathcal{H}_{K},\langle\cdot, \cdot\rangle_{\mathcal{H}_{K}}\right)$ é isometricamente isomorfo a $\left(\mathcal{H}_{\Phi},\langle\cdot, \cdot\rangle_{\mathcal{H}_{\Phi}}\right)$, onde

$$
\mathcal{H}_{\Phi}=\left\{\Phi_{u}: u \in \mathcal{W}\right\}
$$

$e$

$$
\left\langle\Phi_{u}, \Phi_{v}\right\rangle_{\mathcal{H}_{\Phi}}=\langle v, u\rangle_{\mathcal{W}}, \quad \Phi_{u}, \Phi_{v} \in \mathcal{H}_{\Phi}
$$

Demonstração: O primeiro passo será mostrar que o espaço $\mathcal{H}_{\Phi}$ é um espaço de Hilbert. Um cálculo direto revela que

$$
\Phi_{u}+\Phi_{v}=\Phi_{u+v}, \quad u, v \in \mathcal{W}
$$

e

$$
\lambda \Phi_{u}=\Phi_{\bar{\lambda} u} \quad u, v \in \mathcal{W}, \quad \lambda \in \mathbb{C} .
$$

Logo, $\mathcal{H}_{\Phi}$ tem estrutura de espaço vetorial. Observemos ainda que, sendo $\langle\cdot, \cdot\rangle_{\mathcal{W}}$ produto interno em $\mathcal{W}$, a forma $\langle\cdot, \cdot\rangle_{\mathcal{H}_{\Phi}}$ define um produto interno para o espaço $\mathcal{H}_{\Phi}$, como pode ser facilmente verificado. Mostraremos agora que $\mathcal{H}_{\Phi}$ munido do produto (1.4) é um espaço de Hilbert. Para isso notemos que, se $\left(\Phi_{u_{n}}\right), u_{n} \in \mathcal{W}$, é uma sequência de Cauchy neste espaço, então

$$
\begin{aligned}
\left\|\Phi_{u_{n}}-\Phi_{u_{m}}\right\|_{\mathcal{H}_{\Phi}}^{2} & =\left\langle\Phi_{u_{n}}, \Phi_{u_{n}}\right\rangle_{\mathcal{H}_{\Phi}}-\left\langle\Phi_{u_{n}}, \Phi_{u_{m}}\right\rangle_{\mathcal{H}_{\Phi}}-\left\langle\Phi_{u_{m}}, \Phi_{u_{n}}\right\rangle_{\mathcal{H}_{\Phi}}+\left\langle\Phi_{u_{m}}, \Phi_{u_{m}}\right\rangle_{\mathcal{H}_{\Phi}} \\
& =\left\langle u_{n}, u_{n}\right\rangle_{\mathcal{W}}-\left\langle u_{m}, u_{n}\right\rangle_{\mathcal{W}}-\left\langle u_{n}, u_{m}\right\rangle_{\mathcal{W}}+\left\langle u_{m}, u_{m}\right\rangle_{\mathcal{W}},
\end{aligned}
$$

ou seja,

$$
\left\|\Phi_{u_{n}}-\Phi_{u_{m}}\right\|_{\mathcal{H}_{\Phi}}^{2}=\left\|u_{n}-u_{m}\right\|_{\mathcal{W}}^{2}, \quad m, n=1,2, \ldots
$$

Logo, $\left(u_{n}\right)$ é uma sequência de Cauchy em $\mathcal{W}$. Como $\mathcal{W}$ é completo, existe então $u$ em $\mathcal{W}$ tal que

$$
\lim _{n \rightarrow \infty}\left\|u_{n}-u\right\|_{\mathcal{W}}=0
$$

Da igualdade

$$
\left\|\Phi_{u_{n}}-\Phi_{u}\right\|_{\mathcal{H}_{\Phi}}^{2}=\left\|u_{n}-u\right\|_{\mathcal{W}}^{2}, \quad n=1,2, \ldots
$$


concluímos que

$$
\lim _{n \rightarrow \infty}\left\|\Phi_{u_{n}}-\Phi_{u}\right\|_{\mathcal{H}_{\Phi}}=0 .
$$

Para finalizar a demonstração, consideremos a aplicação

$$
\sum_{j=1}^{n} c_{j} K_{y_{j}} \in\left[\left\{K_{y}: y \in E\right\}\right] \stackrel{\zeta}{\longrightarrow} \sum_{j=1}^{n} c_{j} \Phi_{\Phi\left(y_{j}\right)} \in \mathcal{H}_{\Phi}
$$

Utilizando (1.3) e as duas fórmulas citadas no início da demonstração, podemos verificar sem dificuldades que $\zeta$ é linear e injetora. Por outro lado,

$$
\left\langle\zeta\left(K_{x}\right), \zeta\left(K_{y}\right)\right\rangle_{\mathcal{H}_{\Phi}}=\left\langle\Phi_{\Phi(x)}, \Phi_{\Phi(y)}\right\rangle_{\mathcal{H}_{\Phi}}=\langle\Phi(y), \Phi(x)\rangle_{\mathcal{W}}=K(y, x), \quad x, y \in E,
$$

ou seja,

$$
\left\langle\zeta\left(K_{x}\right), \zeta\left(K_{y}\right)\right\rangle_{\mathcal{H}_{\Phi}}=\left\langle K_{x}, K_{y}\right\rangle_{\mathcal{H}_{K}}, \quad x, y \in E .
$$

Da linearidade de $\zeta$ e do produto interno, concluímos que

$$
\langle\zeta(f), \zeta(g)\rangle_{\mathcal{H}_{\Phi}}=\langle f, g\rangle_{\mathcal{H}_{K}}, \quad f, g \in\left[\left\{K_{y}: y \in E\right\}\right] .
$$

Por fim, sendo a imagem de $\Phi$ fundamental em $\mathcal{W}$, segue que $\zeta\left(\left[\left\{K_{y}: y \in E\right\}\right]\right)$ é denso em $\mathcal{H}_{\Phi}$. Todos estes argumentos juntos implicam que $\mathcal{H}_{\Phi}$ é um completamento de $\left[\left\{K_{y}: y \in E\right\}\right]$. Assim, pela unicidade do mesmo garantida pelo Lema 1.2.2, segue que os espaços de Hilbert $\mathcal{H}_{\Phi}$ e $\mathcal{H}_{K}$ são isometricamente isomorfos.

Fechando os argumentos da proposição anterior, observe que o espaço de Hilbert construído tem, de fato, a propriedade de reprodução para $K$ :

$$
\left\langle\Phi_{u}, K_{y}\right\rangle_{\mathcal{H}}=\left\langle\Phi_{u}, \Phi_{\Phi(y)}\right\rangle_{\mathcal{H}}=\langle\Phi(y), u\rangle_{\mathcal{W}}=\Phi_{u}(y), \quad y \in E
$$

A próxima proposição explica com mais detalhes o efeito real da hipótese de fundamentalidade utilizada na proposição anterior. Essencialmente, a proposição descreve como tal hipótese pode ser retirada e a qual preço.

Lembremos que se $F$ é um subespaço fechado de um espaço de Hilbert $\mathcal{H}$, temos a decomposição $\mathcal{H}=F \oplus F^{\perp}$ ([26, p.40]). Para o resultado a seguir, fixada uma função layout $\Phi: E \rightarrow \mathcal{W}$ para $K$, consideraremos a decomposição $\mathcal{W}=\overline{[\Phi(E)]} \oplus \overline{[\Phi(E)]^{\perp}} \mathrm{e}$ a projeção ortogonal $P_{\Phi}$ de $\mathcal{W}$ sobre $\overline{[\Phi(E)]}$.

Proposição 1.3.3. Seja $\Phi: E \rightarrow \mathcal{W}$ uma função layout para o núcleo $K$. Então, $\left(\mathcal{H}_{K},\langle\cdot, \cdot\rangle_{\mathcal{H}_{K}}\right)$ é isometricamente isomorfo a $\left(\mathcal{H},\langle\cdot, \cdot\rangle_{\mathcal{H}}\right)$ onde

$$
\mathcal{H}=\left\{\Phi_{u}: u \in \overline{[\Phi(E)]}\right\}
$$

$e$

$$
\left\langle\Phi_{u}, \Phi_{v}\right\rangle_{\mathcal{H}}=\left\langle P_{\Phi}(v), P_{\Phi}(u)\right\rangle_{\mathcal{W}}, \quad \Phi_{u}, \Phi_{v} \in \mathcal{H}
$$


Demonstração: Como no teorema anterior, $\mathcal{H}$ tem estrutura de espaço vetorial. Para verificar que $\langle\cdot, \cdot\rangle_{\mathcal{H}}$ define um produto interno em $\mathcal{H}$, é suficiente usar a linearidade do produto interno em $\mathcal{W}$ e a linearidade da projeção $P_{\Phi}$. Para a verificação de que $\left(\mathcal{H},\langle\cdot, \cdot\rangle_{\mathcal{H}}\right)$ é um espaço de Hilbert, basta observar que se $\left(\Phi_{u_{n}}\right), u_{n} \in \mathcal{W}$, é uma sequência de Cauchy em $\mathcal{H}$, então

$$
\left\|\Phi_{u_{n}}-\Phi_{u_{m}}\right\|_{\mathcal{H}}^{2}=\left\|u_{n}-u_{m}\right\|_{\mathcal{W}}^{2}=\left\|P_{\Phi}\left(u_{n}\right)-P_{\Phi}\left(u_{m}\right)\right\|_{\mathcal{W}}^{2}, \quad m, n=1,2, \ldots,
$$

e repetir os argumentos da demonstração anterior. Aqui estamos usando o fato da projeção ortogonal ser um operador de norma 1. Como na proposição anterior, a aplicação

$$
\sum_{j=1}^{n} c_{j} K_{y_{j}} \in\left[\left\{K_{y}: y \in E\right\}\right] \stackrel{\zeta}{\longrightarrow} \sum_{j=1}^{n} c_{j} \Phi_{\Phi\left(y_{j}\right)} \in \mathcal{H}
$$

é linear e injetora. Uma vez que a imagem de $P_{\Phi}$ é o conjunto dos pontos fixos de $P_{\Phi}$,

$$
\begin{aligned}
\left\langle\zeta\left(K_{x}\right), \zeta\left(K_{y}\right)\right\rangle_{\mathcal{H}} & =\left\langle\Phi_{\Phi(x)}, \Phi_{\Phi(y)}\right\rangle_{\mathcal{H}} \\
& =\left\langle P_{\Phi}(\Phi(y)), P_{\Phi}(\Phi(x))\right\rangle_{\mathcal{W}} \\
& =\langle\Phi(y), \Phi(x)\rangle_{\mathcal{W}}=K(y, x), \quad x, y \in E .
\end{aligned}
$$

Logo, como na prova da proposição anterior, segue que

$$
\langle\zeta(f), \zeta(g)\rangle_{\mathcal{H}}=\langle f, g\rangle_{\mathcal{H}_{K}}, \quad f, g \in\left[\left\{K_{y}: y \in E\right\}\right] .
$$

Finalmente, como $\overline{[\Phi(E)]}$ é um subespaço fechado do espaço de Hilbert $\mathcal{W}$, ele próprio é um espaço de Hilbert munido do produto interno induzido de $\mathcal{W}$ ([15, p.140]). Essa é a informação primordial na justificativa de que a imagem de $\zeta$ é densa em $\mathcal{H}$.

Observação 1.3.4. Como consequência da desigualdade de Cauchy-Schwarz temos que uma função layout para $K$ satisfaz

$$
\begin{aligned}
\left|\Phi_{u}(x)-\Phi_{u}(y)\right| & =\left|\langle\Phi(x)-\Phi(y), u\rangle_{\mathcal{W}}\right| \\
& \leq\|u\|_{\mathcal{W}}\|\Phi(x)-\Phi(y)\|_{\mathcal{W}}=\left\|\Phi_{u}\right\|_{\mathcal{H}_{K}}\|\Phi(x)-\Phi(y)\|_{\mathcal{W}}, \quad x, y \in E .
\end{aligned}
$$

Se $\Phi$ é contínua (ou equivalentemente, $K$ é contínuo), então cada $\Phi_{u}$ é contínua. A Proposição 1.3.2 nos revela, então, que todo elemento de $\mathcal{H}_{K}$ é uma função contínua, ratificando de maneira alternativa metade do que foi justificado na Proposição 1.2.7.

\subsection{Espaços de Hilbert de Reprodução de dimensão fi- nita}

Nesta última seção do capítulo, estudaremos um pouco mais a fundo os EHR que possuem dimensão finita, incorporando a matriz $A$ da equivalência do Teorema 1.2.6 aos resultados da seção anterior. 
Seja, então, $K$ um núcleo que possui a descrição 1.2 para alguma matriz $A$ de ordem $d$ e funções $\phi_{k}, k=1,2, \ldots, d$, nas condições do teorema. A ideia principal é tentar vizualizar o espaço $\left(\mathcal{H}_{K},\langle\cdot, \cdot\rangle_{\mathcal{H}_{K}}\right)$ através de $A$.

Inicialmente, definamos o produto interno

$$
\langle z, w\rangle_{A}=z A w^{*}=\sum_{i, j=1}^{d} z_{i} \overline{w_{j}} a_{i j}, \quad z, w \in \mathbb{C}^{d},
$$

onde estamos escrevendo $z=\left(z_{1}, z_{2}, \ldots, z_{d}\right)$ e $w=\left(w_{1}, w_{2}, \ldots, w_{d}\right)$. Como $A$ é positiva definida e hermitiana, as propriedades que comprovam que tal aplicação é, de fato, um produto interno em $\mathbb{C}^{d}$ são facilmente verificadas. Definindo, então, $\mathcal{W}:=\left(\mathbb{C}^{d},\langle\cdot, \cdot\rangle_{A}\right)$, é fácil ver que $\Phi: E \rightarrow \mathcal{W}$ dada por

$$
\Phi(x):=\phi(x)=\left(\phi_{1}(x), \phi_{2}(x), \ldots, \phi_{d}(x)\right), \quad x \in E,
$$

é uma função layout para $K$.

Para $c \in \overline{[\Phi(E)]}$, escrevamos $\left(A c^{*}\right)_{i}$ para denotar a $i$-ésima componente do vetor $A c^{*}$.

Teorema 1.4.1. Seja $K$ um núcleo conforme explicitado acima. Então $\mathcal{H}_{K}$ é isometricamente isomorfo ao espaço

$$
\mathcal{H}=\left\{\sum_{i=1}^{d}\left(A c^{*}\right)_{i} \phi_{i}: c=\left(c_{1}, c_{2}, \ldots, c_{d}\right) \in \overline{[\Phi(E)]}\right\} .
$$

munido do produto interno

$$
\left\langle\sum_{i=1}^{d}\left(A b^{*}\right)_{i} \phi_{i}, \sum_{j=1}^{d}\left(A c^{*}\right)_{j} \phi_{j}\right\rangle_{\mathcal{H}_{A}}=P_{\Phi}(c) A P_{\Phi}(b)^{*}, \quad b, c \in \mathbb{C}^{d},
$$

onde $b=\left(b_{1}, b_{2}, \ldots, b_{d}\right)$ e $c=\left(c_{1}, c_{2}, \ldots, c_{d}\right)$.

Demonstração: Aplicando a Proposição 1.3.3, temos que $\mathcal{H}_{K}$ é uma cópia isométrica do espaço

$$
\mathcal{H}=\left\{\Phi_{c}: c=\left(c_{1}, c_{2}, \ldots, c_{d}\right) \in \overline{[\Phi(E)]}\right\},
$$

onde $\Phi_{c}(x)=\langle\Phi(x), c\rangle_{A}, x \in E$. Com isso, pela fórmula do produto interno em questão, obtemos

$$
\mathcal{H}=\left\{\sum_{i, j=1}^{d} a_{i j} \overline{c_{j}} \phi_{i}: c=\left(c_{1}, c_{2}, \ldots, c_{d}\right) \in \overline{[\Phi(E)]}\right\} .
$$

Ou seja,

$$
\mathcal{H}=\left\{\sum_{i=1}^{d}\left(\sum_{j=1}^{d} a_{i j} \overline{c_{j}}\right) \phi_{i}: c=\left(c_{1}, c_{2}, \ldots, c_{d}\right) \in \overline{[\Phi(E)]}\right\} .
$$


Simplificando um pouco mais chegamos a

$$
\mathcal{H}=\left\{\sum_{i=1}^{d}\left(A c^{*}\right)_{i} \phi_{i}: c=\left(c_{1}, c_{2}, \ldots, c_{d}\right) \in \overline{[\Phi(E)]}\right\} .
$$

Para obtermos a fórmula para o produto interno, fixemos dois elementos $f=\sum_{i=1}^{d}\left(A b^{*}\right)_{i} \phi_{i}$ e $g=\sum_{j=1}^{d}\left(A c^{*}\right)_{j} \phi_{j}$ de $\mathcal{H}$. Como

$$
\langle f, g\rangle_{\mathcal{H}}=\left\langle\Phi_{b}, \Phi_{c}\right\rangle_{\mathcal{H}}
$$

a Proposição 1.3 .3 implica que

$$
\langle f, g\rangle_{\mathcal{H}}=\left\langle P_{\Phi}(c), P_{\Phi}(b)\right\rangle_{\mathcal{W}}=P_{\Phi}(c) A P_{\Phi}(b)^{*}
$$

e o resultado segue.

Pequenas modificações nos argumentos utilizados na demonstração do teorema acima produzem a seguinte formulação adicional. No que segue, $A^{T}$ denotará a transposta da matriz $A$.

Corolário 1.4.2. Nas condições do teorema anterior, se a imagem de $\Phi$ é um subconjunto fundamental de $\mathbb{C}^{d}$, então

$$
\mathcal{H}_{K}=\left\{\sum_{i=1}^{d} \psi_{i} w_{i}: w=\left(w_{1}, w_{2}, \ldots, w_{d}\right) \in \mathbb{C}^{d}\right\}
$$

munido do produto interno

$$
\left\langle\sum_{i=1}^{d} \psi_{i} z_{i}, \sum_{j=1}^{d} \psi_{j} w_{j}\right\rangle_{\mathcal{H}_{K}}=\left\langle z\left(A^{T}\right)^{-1}, w\right\rangle_{\mathbb{C}^{d}}, \quad w, z \in \mathbb{C}^{d}
$$

onde $w=\left(w_{1}, w_{2}, \ldots, w_{d}\right)$ e $z=\left(z_{1}, z_{2}, \ldots, z_{d}\right)$. 



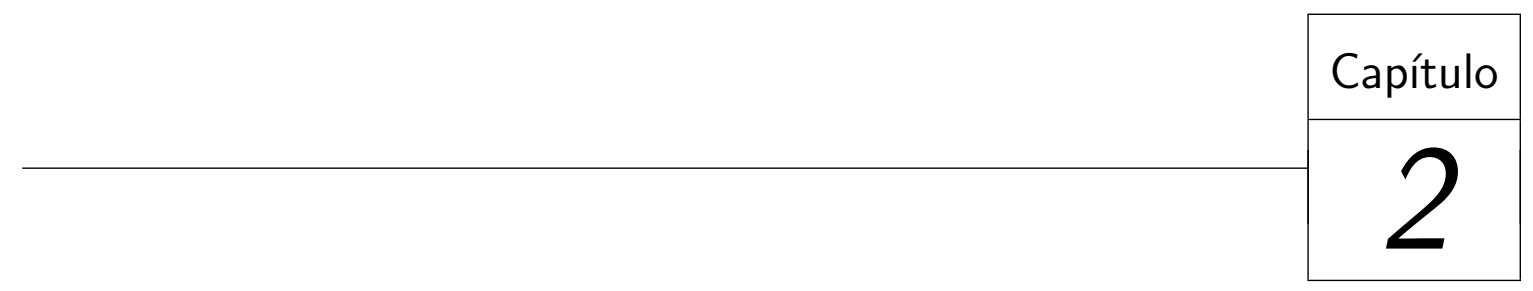

Núcleos universais

Ao longo deste capítulo, $E$ será um espaço topológico de Hausdorff e $K$ um núcleo PD contínuo sobre $E$. Se $X$ é um subconjunto compacto não vazio de $E$, denotaremos por $C(X)$ o espaço de todas as funções contínuas a valores complexos definidas em $X$, munido de sua topologia da convergência uniforme em $X$. A norma em $C(X)$ será denotada por $\|\cdot\|_{\infty}$. Isto feito, diremos que $K$ é um núcleo universal se para cada subconjunto compacto $X$ de $E$, o conjunto $\{K(\cdot, y): y \in X\}$ formado pelas restrições das aplicações $K_{y}$ a $X$ é fundamental em $C(X)$. Equivalentemente, $K$ é universal se, e somente se, fixados o compacto $X$, uma função contínua $f: X \rightarrow \mathbb{C}$ e $\varepsilon>0$, existe uma função $g$ no fecho de $[\{K(\cdot, y): y \in X\}]$ em $C(X)$, de modo que

$$
\|f-g\|_{\infty}:=\max _{x \in X}|f(x)-g(x)|<\varepsilon .
$$

Neste capítulo, estudaremos detalhadamente a universalidade de um núcleo contínuo $K$ em conexão com uma função layout para $K$ fixada. Já que existe uma forte ligação entre o núcleo PD e qualquer uma de suas funções layout, determinaremos que propriedade a função layout deve possuir para que tenhamos garantida a universalidade do núcleo e vice e versa. O processo para se chegar a resultados desta natureza é longo e técnico, razão pela qual dividiremos este capítulo em várias seções.

\subsection{O teorema de Riesz e a função layout}

Fixada uma função layout $\Phi: E \rightarrow \mathcal{W}$, estudaremos nesta seção algumas transformações integrais especiais definidas através de $\Phi$. Os resultados são puramente técnicos 
e entram nas justificativas dos resultados principais do trabalho apresentados à frente. A menos de especificação em contrário, o símbolo $X$ indicará um subconjunto compacto arbitrário e não vazio de $E$.

Escreveremos $\mathcal{M}(X)$ para denotar o espaço de todas as medidas de Radon complexas sobre $X$. Para cada $\mu$ em $\mathcal{M}(X)$, podemos construir naturalmente um elemento do dual $C(X)^{*}$ de $C(X)$ através da fórmula

$$
I_{\mu}(f)=\int_{X} f d \mu, \quad f \in C(X) .
$$

A expressão

$$
\|\mu\|=\sup \left\{\left|I_{\mu}(g)\right|: g \in C(X):\|g\|_{\infty} \leq 1\right\},
$$

é usualmente conhecida por variação total de $\mu$ e define uma norma em $\mathcal{M}(X)$. Isto pode ser ratificado em [11, p.94] ou no Capítulo 5 de [13]. Mais do que isso, segundo o Teorema de Representação de Riesz ([11, p.223]), a aplicação

$$
\mu \in \mathcal{M}(X) \stackrel{\iota}{\longrightarrow} I_{\mu} \in C(X)^{*}
$$

é um isomorfismo isométrico. Por outro lado, se $\chi_{Y}$ denota a função característica de $Y$, a fórmula

$$
|\mu|(Y)=\sup \left\{\left|I_{\mu}\left(g \chi_{Y}\right)\right|: g \in C(X):\|g\|_{\infty} \leq 1\right\}, \quad Y \subseteq X,
$$

define uma medida sobre $X$, a medida de variação total de $\mu$. Sua relação com a variação total de $\mu$ é descrita pela fórmula $\|\mu\|=|\mu|(X)$.

Lema 2.1.1. Sejam $\mu$ um elemento de $\mathcal{M}(X)$ e $K$ um núcleo PD e contínuo sobre $E$. Suponha que $\Phi: E \rightarrow \mathcal{W}$ seja uma função layout para $K$. Então, a fórmula

$$
L(u):=\overline{\int_{X} \Phi_{u}(x) d \mu(x)}, \quad u \in \mathcal{W},
$$

define um elemento de $C(\mathcal{W})^{*}$. Ainda, existe um único $w_{0} \in \mathcal{W}$ de modo que

$$
\overline{L(u)}=\left\langle w_{0}, u\right\rangle_{\mathcal{W}}, \quad u \in \mathcal{W} .
$$

Demonstração: É fácil ver que $L$ é uma aplicação linear. Para verificarmos a sua continuidade, primeiro note que

$$
|L(u)| \leq\left|\int_{X} \Phi_{u}(x) d \mu(x)\right| \leq \int_{X}\left|\langle\Phi(x), u\rangle_{\mathcal{W}}\right| d|\mu|(x), \quad u \in \mathcal{W} .
$$

Usando a desigualdade de Cauchy-Schwarz, concluímos que

$$
|L(u)| \leq \int_{X}\|\Phi(x)\|_{\mathcal{W}}\|u\|_{\mathcal{W}} d|\mu|(x), \quad u \in \mathcal{W} .
$$


Como $\Phi$ é contínua,

$$
|L(u)| \leq \sup _{x \in X}\|\Phi(x)\|_{\mathcal{W}} \int_{X}\|u\|_{\mathcal{W}} d|\mu|(x), \quad u \in \mathcal{W} .
$$

Como medidas de Radon complexas são regulares, segue que

$$
\|L\| \leq \sup _{x \in X}\|\Phi(x)\|_{\mathcal{W}}\|\mu\|<\infty
$$

A afirmação do lema é, então, uma consequência do Teorema da Representação de Riesz usual.

O elemento $w_{0}$ fornecido pelo lema anterior depende de $X, \Phi$ (portanto, de $K$ ) e $\mu$. Logo, se um destes dados está devidamente fixado, esta dependência, apesar de ainda existir, poderia ser omitida nas notações. Sempre omitiremos a dependência de $X$, já que o contexto deixará isto bem claro.

Nas condições do lema anterior, denotaremos por $w_{\Phi}^{\mu}$ o único elemento $w_{0}$ lá descrito. Em outras palavras, $w_{\Phi}^{\mu}$ é o único elemento de $\mathcal{W}$ que satisfaz

$$
\left\langle w_{\Phi}^{\mu}, u\right\rangle_{\mathcal{W}}=\int_{X} \Phi_{u}(x) d \mu(x), \quad u \in \mathcal{W} .
$$

A seguinte consequência da fórmula acima será utilizada à frente.

Corolário 2.1.2. Seja $\mu$ um elemento de $\mathcal{M}(X)$. Suponha que $\Phi: E \rightarrow \mathcal{W}$ seja uma função layout para K. Então,

$$
\left\langle w_{\Phi}^{\mu}, \Phi(y)\right\rangle_{\mathcal{W}}=\int_{X} K(x, y) d \mu(x), \quad y \in X
$$

Demonstração: É suficiente observar que a imagem de $\Phi$ é um subconjunto de $\mathcal{W}$ e utilizar a fórmula da definição anterior com $u=\Phi(y), y \in X$.

$\mathrm{Na}$ proposição abaixo computaremos a norma de $w_{\Phi}^{\mu}$ em $\mathcal{W}$, quando $\mu$ e $\Phi$ estão fixados. O símbolo $\bar{\mu}$ indicará a medida conjugada da medida $\mu$ de $\mathcal{M}(X)$.

Proposição 2.1.3. Seja $\mu$ um elemento de $\mathcal{M}(X)$. Suponha que $\Phi: E \rightarrow \mathcal{W}$ seja uma função layout para K. Então,

$$
\left\|w_{\Phi}^{\mu}\right\|_{\mathcal{W}}^{2}=\int_{X}\left(\int_{X} K(x, y) d \bar{\mu}(y)\right) d \mu(x)
$$

Demonstração: Conjugando a equação (2.4) e usando o fato de $K$ ser hermitiano, vemos que

$$
\overline{\left\langle w_{\Phi}^{\mu}, \Phi(y)\right\rangle_{\mathcal{W}}}=\int_{X} K(y, x) d \bar{\mu}(x), \quad y \in X
$$


Integrando membro a membro em relação a $\mu$ e utilizando 1.3$)$ e 2.3 vem que

$$
\int_{X}\left(\int_{X} K(y, x) d \bar{\mu}(x)\right) d \mu(y)=\int_{X}\left\langle\Phi(y), w_{\Phi}^{\mu}\right\rangle_{\mathcal{W}} d \mu(y)=\left\langle w_{\Phi}^{\mu}, w_{\Phi}^{\mu}\right\rangle_{\mathcal{W}}=\left\|w_{\Phi}^{\mu}\right\|_{\mathcal{W}}^{2} .
$$

Isto completa a demonstração.

No último resultado desta seção registraremos duas propriedades da aplicação $j_{X}: \mathcal{M}(X) \rightarrow \mathcal{W}$ dada por

$$
j_{X}(\mu)=w_{\Phi}^{\mu}, \quad \mu \in \mathcal{M}(X)
$$

Proposição 2.1.4. Seja $\mu$ um elemento de $\mathcal{M}(X)$. Suponha que $\Phi: E \rightarrow \mathcal{W}$ seja uma função layout para $K$. Então, a aplicação $j_{X}$ é linear e contínua.

Demonstração: A linearidade segue de 2.3 e da unicidade de $w_{\Phi}^{\mu}$. Para obtermos a limitação da aplicação note que, usando a última igualdade na demonstração da proposição anterior, deduzimos que

$$
\left\|w_{\Phi}^{\mu}\right\|_{\mathcal{W}}^{2} \leq \int_{X} \int_{X}|K(x, y)| d|\mu|(y) d|\mu|(x) .
$$

Portanto,

$$
\left\|w_{\Phi}^{\mu}\right\|_{\mathcal{W}}^{2} \leq \max _{x, y \in X}|K(x, y)|(|\mu|(X))^{2}=\max _{x, y \in X}|K(x, y)|\|\mu\|^{2},
$$

ou seja, $j_{X}$ é limitada e sua norma não excede $\max _{x, y \in X}|K(x, y)|$.

Observação 2.1.5. A eliminação dos parênteses na primeira desigualdade da demonstração acima é justificada pelo Teorema de Fubini-Tonelli ([11, p.67]).

\subsection{A injetividade de $j_{X}$}

Nesta seção, $X$ ainda será um subconjunto compacto não vazio de $E$. Coletaremos vários resultados envolvendo as aplicações $\iota$ e $j_{X}$ definidas anteriormente. Estaremos particularmente interessados em possíveis relações entre elas e uma caracterização para a injetividade de $j_{X}$.

Se $U$ é um subconjunto de um espaço de Hilbert, a linearidade e continuidade do produto interno do espaço fornecem a igualdade $\overline{[U]}^{\perp}=U^{\perp}$. Escreveremos $U^{\perp \perp}$ para denotar o subespaço $\left(U^{\perp}\right)^{\perp}$. Se $U$ é um subespaço fechado do espaço de Hilbert, então é sabido que $U^{\perp \perp}=U([26$, p.41]).

No primeiro resultado da seção descreveremos o fecho da imagem de $j_{X}$ em $\mathcal{W}$.

Proposição 2.2.1. Se $\Phi: E \rightarrow \mathcal{W}$ é uma função layout para $K$, então os fechos da imagem de $j_{X}$ e do gerado pela imagem de $\Phi$ em $\mathcal{W}$ coincidem, isto é, $\overline{j_{X}(\mathcal{M}(X))}=\overline{[\Phi(X)]}$. 
Demonstração: Fixemos uma função layout $\Phi: E \rightarrow \mathcal{W}$ para $K$. A demonstração consiste em justificar que $j_{X}(\mathcal{M}(X))^{\perp}=\Phi(X)^{\perp}$ e, então, usar as propriedades mencionadas no parágrafo que antecede a proposição. Se $u \in \Phi(X)^{\perp}$, podemos usar a equação (2.3) para obter

$$
\left\langle j_{X}(\mu), u\right\rangle_{\mathcal{W}}=\left\langle w_{\Phi}^{\mu}, u\right\rangle_{\mathcal{W}}=\int_{X} \Phi_{u}(x) d \mu(x)=0, \quad \mu \in \mathcal{M}(X),
$$

e concluir que $u \in j_{X}(\mathcal{M}(X))^{\perp}$. Reciprocamente, se $v \in j_{X}(\mathcal{M}(X))^{\perp}$, um procedimento análogo nos permite concluir que

$$
\int_{X} \Phi_{v}(x) d \mu(x)=0, \quad \mu \in \mathcal{M}(X) .
$$

Agora, para cada $x \in X$, a medida de Dirac $\delta_{x}$ é um elemento de $\mathcal{M}(X)$. Usando a igualdade acima para cada $\delta_{x}$, concluímos que $\langle\Phi(x), v\rangle_{\mathcal{W}}=\Phi_{v}(x)=0, x \in X$. Portanto, $v \in \Phi(X)^{\perp}$. Tendo provado a igualdade inicial, podemos agora escrever

$$
{\overline{j_{X}(\mathcal{M}(X))^{\perp}}}^{\perp}=j_{X}(\mathcal{M}(X))^{\perp}=\Phi(X)^{\perp}=\overline{[\Phi(X)}^{\perp} .
$$

Tomando-se o ortogonal em ambos os membros da igualdade acima, temos que

$$
\overline{j_{X}(\mathcal{M}(X))}={\overline{j_{X}(\mathcal{M}(X))}}^{\perp \perp}=\overline{[\Phi(X)}^{\perp \perp}=\overline{[\Phi(X)]} .
$$

Isto completa a demonstração.

A seguir, lidaremos formalmente com os anuladores de alguns subconjuntos de $C(X)$ e também com suas imagens inversas pelo isomorfismo $\iota$. Se $A$ é um subconjunto de $C(X)$, o anulador de $A$ é o subespaço $A^{o}$ de $C(X)^{*}$ dado por

$$
A^{o}:=\left\{F \in C(X)^{*}: F(f)=0, f \in A\right\} .
$$

Consequentemente,

$$
\iota^{-1}\left(A^{o}\right)=\left\{\mu \in \mathcal{M}(X): \iota(\mu) \in A^{o}\right\}=\left\{\mu \in \mathcal{M}(X): \int_{X} f(x) d \mu(x)=0, f \in A\right\} .
$$

Os dois lemas a seguir descrevem propriedades técnicas que até extrapolam o contexto no qual estamos trabalhando. A primeira delas é usada implicitamente em algumas passagens à frente.

Lema 2.2.2. Se $A$ é um subconjunto de $C(X)$, então $\iota^{-1}\left(A^{o}\right)=\iota^{-1}\left(\overline{[A]}^{o}\right)$.

Demonstração: Fixado um subconjunto $A$ de $C(X)$, é suficiente demonstramos que $A^{o}=\overline{[A]}^{o}$. Como $A \subset[A]$, a inclusão $\overline{[A]}^{o} \subset A^{o}$ é óbvia. Reciprocamente, se $F \in C(X)^{*}$ anula todas as funções de $A$, então $F$ também anula todas as funções de $[A]$ por linearidade. Como $\mathrm{F}$ é uma função contínua, então $F$ anula todas as funções em $\overline{[A]}$. Em outras palavras, a inclusão $A^{o} \subset \overline{[A]}^{o}$ também vale. 
Lema 2.2.3. Sejam $A$ e $B$ subconjuntos de $C(X)$. Então, $\iota^{-1}\left(A^{o}\right)=\iota^{-1}\left(B^{o}\right)$ se, $e$ somente se, $\overline{[A]}=\overline{[B]}$.

Demonstração: Se $\overline{[A]}=\overline{[B]}$, então uma aplicação direta do lema anterior implica que $\iota^{-1}\left(A^{o}\right)=\iota^{-1}\left(B^{o}\right)$. Reciprocamente, suponha que $\iota^{-1}\left(A^{o}\right)=\iota^{-1}\left(B^{o}\right)$, mas que $\overline{[A]} \neq \overline{[B]}$. Sem perda de generalidade, assumiremos que $\overline{[A]} \varsubsetneqq \overline{[A]} \cup \overline{[B]}$. Como $\overline{[A]}$ é fechado em $\overline{[A]} \cup \overline{[B]}$, por uma das consequências do Teorema de Hahn-Banach ([11, p.159]) existe $F \in C(\overline{[A]} \cup \overline{[B]})^{*}$ que anula $\overline{[A]}$ mas não anula $\overline{[A]} \cup \overline{[B]} \backslash \overline{[A]}$. Logo, $F$ anula $\overline{[A]}$ mas não anula $\overline{[B]} \backslash \overline{[A]}$. Usando o Teorema de Hahn-Banach e o fato de $\overline{[A]} \cup \overline{[B]}$ ser fechado em $C(X)$, podemos obter uma extensão (conveniente) $\tilde{F}$ de $F$ em $C(X)^{*}$. Escrevamos, via 2.1), $\tilde{F}=I_{\mu}$, para alguma $\mu \in M(X)$. Então, $\mu \in \iota^{-1}\left(A^{o}\right)$ mas $\mu \notin \iota^{-1}\left(B^{o}\right)$. Isto contradiz nossa suposição inicial.

A seguinte proposição é agora evidente.

Proposição 2.2.4. Sejam $A$ e $B$ subespaços fechados de $C(X)$. Então, $A=B$ se, e somente se, $\iota^{-1}\left(A^{o}\right)=\iota^{-1}\left(B^{o}\right)$.

O resultado a seguir é uma consequência direta do Teorema de Hahn-Banach.

Lema 2.2.5. Seja $A$ um subconjunto de $C(X)$. Então $\overline{[A]}=C(X)$ se, e somente se, $\iota^{-1}\left(A^{o}\right)=\{0\}$.

Demonstração: O Teorema de Hahn-Banach garante que $\overline{[A]}=C(X)$ se, e somente se, $A^{o}=\{0\}$. A equivalência entre esta última igualdade e $\iota^{-1}\left(A^{o}\right)=\{0\}$ é imediata.

Tendo estabelecido os resultados técnicos necessários, concluiremos a seção com um resultado que descreve uma equivalência para a injetividade de $j_{X}$. Antes disso, porém, introduziremos notações adicionais. Escreveremos $G_{K}(X)$ para indicar o espaço de todas as funções em $C(X)$ que são limites uniformes (de restrições a $X$ ) de funções da forma

$$
\sum_{k=1}^{n} c_{k} K\left(\cdot, x_{k}\right), \quad x_{1}, x_{2}, \ldots, x_{n} \in X, \quad n=1,2, \ldots
$$

Em outras palavras,

$$
G_{K}(X)=\overline{\left[\left\{K_{y}: y \in X\right\}\right]},
$$

onde o fecho acima é tomado em $C(X)$.

Teorema 2.2.6. Seja $\Phi: E \rightarrow \mathcal{W}$ uma função layout para K. Então

$$
\operatorname{ker} j_{X}=\left\{\mu \in \mathcal{M}(X): \iota(\mu) \in G_{K}(X)^{o}\right\}=\iota^{-1}\left(G_{K}(X)^{o}\right) \text {. }
$$

Consequentemente, $j_{X}$ é injetora se, e somente se, $G_{K}(X)=C(X)$. 
Demonstração: Se $\mu \in i^{-1}\left(G_{K}(X)^{o}\right) \stackrel{\text { Lema }}{=} \iota^{2.2 .2} \iota^{-1}\left(\left\{K_{y}: y \in X\right\}^{o}\right)$, então

$$
0=\int_{X} K_{y}(x) d \mu(x)=\int_{X} K(x, y) d \mu(x), \quad y \in X .
$$

Neste ponto entramos com a função layout para $K$. Lembrando a Proposição 2.1.3. a definição da aplicação $j_{X}$ e usando o Teorema de Fubini para inverter a ordem de integração, obtemos

$$
\left\|j_{X}(\mu)\right\|_{\mathcal{W}}^{2}=\left\|w_{\Phi}^{\mu}\right\|_{\mathcal{W}}^{2}=\int_{X}\left(\int_{X} K(x, y) d \mu(x)\right) d \bar{\mu}(y)=\int_{X} 0 d \bar{\mu}(y)=0,
$$

ou seja, $\mu \in \operatorname{ker} j_{X}$. Isto mostra que $\iota^{-1}\left(G_{K}(X)^{o}\right) \subset \operatorname{ker} j_{X}$. Reciprocamente, se $\nu \in \mathcal{M}(X)$ e $j_{X}(\nu)=0$, podemos usar a fórmula (2.4) para deduzir que

$$
\int_{X} K(x, y) d \nu(x)=\left\langle j_{X}(\nu), \Phi(y)\right\rangle_{\mathcal{W}}=0, \quad y \in X
$$

ou seja,

$$
\int_{X} K_{y}(x) d \nu(x)=0, \quad y \in X
$$

Logo, $\nu \in \iota^{-1}\left(\left\{K_{y}: y \in X\right\}^{o}\right)=\iota^{-1}\left(G_{K}(X)^{o}\right)$ e, portanto, ker $j_{X} \subset \iota^{-1}\left(G_{K}(X)^{o}\right)$. Para justificarmos a última parte do enunciado do teorema, note que a injetividade de $j_{X}$ é agora equivalente à igualdade $i^{-1}\left(G_{K}(X)^{o}\right)=\{0\}$. No entanto, pelo Lema 2.2.5. ela torna-se equivalente à igualdade $C(X)=\overline{\left[G_{K}(X)\right]}$. Como $G_{K}(X)$ já é fechado em $C(X)$, esta igualdade é, de fato, $C(X)=G_{K}(X)$.

\subsection{A aplicação $l_{X}$ e o seu papel}

Nesta seção, ainda manteremos um compacto não vazio $X$ de $E$ fixo e a continuidade do núcleo $K$.

Fixada uma função layout $\Phi: E \rightarrow \mathcal{W}$, estudaremos o papel da transformação conjugada linear $l_{X}: \mathcal{W} \rightarrow C(X)$ dada por

$$
l_{X}(u)=\Phi_{u}, \quad u \in \mathcal{W} .
$$

A expressão que define $l_{X}$ é exatamente aquela que aparece no integrando da fórmula (2.3) que estabelece a definição do elemento $w_{\Phi}^{u}$ oriundo da aplicação layout $\Phi$. Ela é útil principalmente em descrições alternativas para o conjunto $G_{K}(X)$.

Note que para tal aplicação verifica-se a igualdade

$$
\left\langle j_{X}(\mu), u\right\rangle_{\mathcal{W}}=\int_{X} l_{X}(u)(x) d \mu(x)=I_{\mu}\left(l_{X}(u)\right), \quad \mu \in M(X), \quad u \in \mathcal{W} .
$$

Vejamos agora a descrição de $G_{K}(X)$ através de $l_{X}$. 
Teorema 2.3.1. Se $\Phi: E \rightarrow \mathcal{W}$ é uma função layout para $K$, então $G_{K}(X)$ coincide com o fecho da imagem de $l_{X}$ em $C(X)$, isto é, $G_{K}(X)=\overline{l_{X}(\mathcal{W})}$.

Demonstração: Seja $\Phi: E \rightarrow \mathcal{W}$ uma função layout para $K$. Ambos os espaços da igualdade do enunciado do teorema são fechados em $C(X)$. Logo, devido a Proposição 2.2.4, o teorema estará demonstrado tão logo provemos que a igualdade $\iota^{-1}\left(G_{K}(X)^{o}\right)=\iota^{-1}\left({\overline{l_{X}(\mathcal{W})}}^{o}\right)$ vale. Pelo Teorema 2.2.6, já temos que $\iota^{-1}\left(G_{K}(X)^{o}\right)=$ ker $j_{X}$, enquanto que o Lema 2.2 .2 nos dá que $\iota^{-1}\left(\overline{\bar{l}_{X}(\mathcal{W})^{o}}\right)=\iota^{-1}\left(l_{X}(\mathcal{W})^{o}\right)$. Portanto, a demonstração resume-se a mostrar que ker $j_{X}=\iota^{-1}\left(l_{X}(\mathcal{W})^{o}\right)$. Mas, usando (2.7), vemos imediatamente que para $\mu \in \mathcal{M}(X)$ fixada, a condição $j_{X}(\mu)=0$ equivale a $\iota(\mu)\left(l_{X}(u)\right)=0, u \in \mathcal{W}$, ou seja, equivale a $\iota(\mu) \in l_{X}(\mathcal{W})^{o}$. A demonstração está completa.

Corolário 2.3.2. Seja $\Phi: E \rightarrow \mathcal{W}$ uma função layout para $K$. Se U é um subconjunto fundamental de $\mathcal{W}, l_{X}(U)$ é fundamental em $G_{K}(X)$. Em outras palavras, se $\overline{[U]}=\mathcal{W}$, então $G_{K}(X)=\overline{\left[l_{X}(U)\right]}$.

Demonstração: Seja $U \subset \mathcal{W}$ tal que $\overline{[U]}=\mathcal{W}$. Pelo teorema anterior temos que

$$
G_{K}(X)=\overline{l_{X}(\overline{[U]})}=\overline{\overline{l_{X}([U])}}=\overline{\overline{\left[l_{X}(U)\right]}}=\overline{\left[l_{X}(U)\right]},
$$

e o resultado segue.

A última caracterização para $G_{K}(X)$ que apresentaremos, dependerá de uma base ortonormal para o espaço de Hilbert $\mathcal{W}$ que aparece na definição de função layout. Como $\mathcal{W}$ pode não ser separável, lembramos dois aspectos fundamentais de uma base ortonormal $\mathcal{B}$ para $\mathcal{W}$ : fixado $u \in \mathcal{W}$, o conjunto

$$
\left\{v \in \mathcal{B}:\langle u, v\rangle_{\mathcal{W}} \neq 0\right\}
$$

é enumerável. Além disso, vale a decomposição

$$
u=\sum_{v \in \mathcal{B}}\langle u, v\rangle v
$$

com convergência da série, qualquer que seja a ordenação em $\mathcal{B}$. Tais resultados podem ser ratificados em [2, p.144].

Se $\Phi: E \rightarrow \mathcal{W}$ é uma função layout para $K$ e uma base ortonormal $\mathcal{B}$ está disponível para $\mathcal{W}$, podemos definir o conjunto

$$
\Phi_{X}(\mathcal{B}):=\overline{\left[\left\{\left.\Phi_{v}\right|_{X}: v \in \mathcal{B}\right\}\right]}
$$

Observação 2.3.3. A similaridade entre as notações $\Phi(X)$ e $\Phi_{X}(\mathcal{B})$ não deve gerar confusão, uma vez que $\Phi(X)$ é um subconjunto de $\mathcal{W}$ e $\Phi_{X}(\mathcal{B})$ é um subconjunto de $C(X)$. 
Estamos prontos para uma terceira caracterização para $G_{K}(X)$.

Teorema 2.3.4. Seja $\Phi: E \rightarrow \mathcal{W}$ uma função layout para $K$. Se $\mathcal{B}$ é uma base ortonormal para $\mathcal{W}$, então $G_{K}(X)=\Phi_{X}(\mathcal{B})$.

Demonstração: Como $\overline{[\mathcal{B}]}=\mathcal{W}$, o Corolário 2.3 .2 implica que

$$
G_{K}(X)=\overline{\left[l_{X}(\mathcal{B})\right]}=\overline{\left[\left\{\left.\Phi_{v}\right|_{X}: v \in \mathcal{B}\right\}\right]}=\Phi_{X}(\mathcal{B})
$$

a igualdade do enunciado.

\subsection{Universalidade}

Nesta seção, apresentaremos os resultados principais do trabalho. Essencialmente, descreveremos resultados que fornecem equivalências para que um núcleo $K \mathrm{PD}$ e contínuo sobre $E$ tenha a propriedade universal da aproximação.

Iniciaremos com a formalização do conceito de universalidade.

Definição 2.4.1. Um núcleo contínuo $K: E \times E \rightarrow \mathbb{C}$ possui a propriedade universal da aproximação (ou resumidamente que é $K$ é um núcleo universal) se para cada subconjunto compacto e não vazio $X$ de $E$, qualquer $\varepsilon>0$ e qualquer função $f \in C(X)$, existe $g \in G_{K}(X)$ tal que

$$
\|f-g\|_{\infty} \leq \varepsilon
$$

Observamos que, devido à definição de $G_{K}(X)$ em (2.6), a universalidade $K$ é equivalente à fundamentalidade de $\left\{K_{y}: y \in X\right\}$ em $C(X)$, qualquer que seja o compacto não vazio $X$ do espaço topológico $E$. Notemos ainda que, conforme indicado no início do capítulo, o conjunto mencionado acima é composto das restrições das funções $K_{y}$ a $X$. Outra maneira de expressar a propriedade de universalidade para um núcleo $K$ é exigir que $G_{K}(X)=C(X)$, qualquer que seja o compacto não vazio $X$ de $E$.

De maneira análoga, introduziremos universalidade para uma função layout $\Phi$ de $K$, via o conjunto $\Phi_{X}(\mathcal{B})$ introduzido na seção anterior.

Definição 2.4.2. Sejam $\Phi: E \rightarrow \mathcal{W}$ uma função layout para um núcleo $K P D$ sobre $E$ e $\mathcal{B}$ uma base ortonormal para o espaço de Hilbert $\mathcal{W}$. A função $\Phi$ é universal se para cada subconjunto compacto e não vazio $X$ de $E$, qualquer $\varepsilon>0$ e qualquer função $f \in C(X)$, existe $g \in \Phi_{X}(\mathcal{B})$ tal que

$$
\|f-g\|_{\infty} \leq \varepsilon
$$


Observamos que a universalidade de $\Phi$ é então equivalente à fundamentalidade do conjunto $\left\{\left.\Phi_{v}\right|_{X}: v \in \mathcal{B}\right\}$ em $\mathrm{C}(\mathrm{X})$, qualquer que seja o subconjunto compacto não vazio $X$ de $E$. Equivalentemente, $\Phi_{X}(\mathcal{B})=C(X)$, qualquer que seja o subconjunto compacto não vazio $X$ de $E$.

Uma primeira caracterização para universalidade do núcleo $K$ está, então, implícita no Teorema 2.3.4.

Teorema 2.4.3. Sejam $\Phi: E \rightarrow \mathcal{W}$ uma função layout para o núcleo K PD e contínuo sobre $E$ e $\mathcal{B}$ uma base ortonormal para o espaço de Hilbert $\mathcal{W}$. Então, $K$ é universal se, e somente se, $\Phi$ é universal.

\subsection{Universalidade e a teoria de Mercer}

A teoria de Mercer pode ser intepretada como aquela que engloba resultados envolvendo simultaneamente um núcleo positivo definido e o operador integral gerado por ele. Nesta seção, analisaremos os conceitos de universalidade introduzidos na seção anterior, envolvendo um pouco da teoria de Mercer nos resultados.

Em toda a seção, $K$ será um núcleo PD contínuo sobre o espaço topológico $E$.

Se $X$ é um subconjunto compacto e não vazio de $E$ e $\mu$ é uma medida de Borel sobre $X$, então a fórmula

$$
\mathcal{K}(g)(x):=\int_{X} K(x, y) g(y) d \mu(y)
$$

define um operador integral $\mathcal{K}: L^{2}(X, \mu) \rightarrow L^{2}(X, \mu)$. A análise funcional clássica garante, então, que a restrição de $K$ a $X \times X$ é representável por uma série absoluta e uniformemente convergente da forma

$$
K(x, y)=\sum_{n=1}^{\infty} \lambda_{n} \phi_{n}(x) \overline{\phi_{n}(y)}, \quad x, y \in X
$$

onde $\left\{\phi_{n}\right\}$ é um conjunto $L^{2}(X, \mu)$-ortonormal formado por funções contínuas, $\phi_{n}$ é uma auto-função do operador integral $\mathcal{K}$ associada ao autovalor $\lambda_{n} \geq 0$, respectivamente, e 0 é o único ponto de acumulação de $\left\{\lambda_{n}\right\}$. Por simplicidade, assumiremos que $\lambda_{n}>0$ para todo $n$.

Até o fnal desta seção, exceto se mencionado o contrário, assumiremos o contexto descrito acima. Consequentemente, sempre consideraremos uma função layout $\Phi: E \rightarrow \mathcal{W}\left(=\ell_{2}\right)$ para $K$ da forma $\Phi(x)=(x(j)), x \in E$, onde

$$
x(j):=\lambda_{j}^{1 / 2} \phi_{j}(x), \quad x \in X, \quad j=0,1, \ldots,
$$


como adiantado no Teorema 1.2.5.

Um primeiro resultado que pode gerar uma equivalência para a universalidade de $K$ no âmbito da teoria de Mercer está descrito abaixo.

Teorema 2.5.1. Se $K$ é um núcleo $P D$ e contínuo como descrito no início da seção, então, $G_{K}(X)=C(X)$ se, e somente se, $\overline{\left[\left\{\phi_{n}: n=1,2, \ldots\right\}\right]}=C(X)$.

Demonstração: Fixado $X$, a ideia da demonstração é aproveitar tanto quanto possível, o Teorema 2.3.4. Para tanto, comecemos notando que

$$
\Phi_{v}(x)=\sum_{j=1}^{\infty} v(j) \lambda_{j}^{1 / 2} \phi_{j}(x), \quad x \in X,
$$

onde estamos escrevendo $v=(v(j)) \in \mathcal{B}$ (base ortonormal de $\ell_{2}$ ). A fórmula acima implica

imediatamente que cada $\left.\Phi_{v}\right|_{X}$ é um elemento de $\overline{\left[\left\{\phi_{j}: j=1,2, \ldots\right\}\right]}$ e, consequentemente,

$$
\Phi_{X}(\mathcal{B}) \subseteq \overline{\left[\left\{\phi_{j}: j=1,2, \ldots\right\}\right]} .
$$

Para demonstrarmos a inclusão reversa, tomemos um elemento genérico

$$
\xi=\sum_{i=1}^{n} \alpha_{i} \phi_{i}
$$

em $\left[\left\{\phi_{j}: j=1,2, \ldots\right\}\right]$. A fórmula

$$
w:=\left(\alpha_{1} \lambda_{1}^{-1 / 2}, \alpha_{2} \lambda_{2}^{-1 / 2}, \ldots, \alpha_{n} \lambda_{n}^{-1 / 2}, 0,0, \ldots\right)
$$

define um elemento de $\ell_{2}$ que satisfaz

$$
\langle\Phi(x), w\rangle_{2}=\sum_{j=1}^{n} \lambda_{j}^{1 / 2} \phi_{j}(x) \alpha_{j} \lambda_{j}^{-1 / 2}=\sum_{j=1}^{n} \alpha_{j} \phi_{j}(x)=\xi(x), \quad x \in X .
$$

Como $\ell_{2}$ é separável, podemos, sem perda de generalidade, escrever $\mathcal{B}=\left\{v_{1}, v_{2}, \ldots\right\}$. Decompondo $w$ com relação a esta base, digamos,

$$
w=\sum_{j=1}^{\infty} \gamma_{j} v_{j}, \quad \gamma_{j} \in \mathbb{C},
$$

obtemos, via continuidade do produto interno,

$$
\xi(x)=\left\langle\Phi(x), \sum_{j=1}^{\infty} \gamma_{j} v_{j}\right\rangle_{2}=\sum_{j=1}^{\infty} \overline{\gamma_{j}}\left\langle\Phi(x), v_{j}\right\rangle_{2}=\sum_{j=1}^{\infty} \overline{\gamma_{j}} \Phi_{v_{j}}(x), \quad x \in X .
$$


Segue que $\xi \in \overline{\left[\left\{\left.\Phi_{v}\right|_{X}: v \in \mathcal{B}\right\}\right]}=\Phi_{X}(\mathcal{B})$ e, consequentemente, $\overline{\left[\left\{\phi_{j}: j=1,2, \ldots\right\}\right]} \subseteq$ $\Phi_{X}(\mathcal{B})$. Assim, $\overline{\left[\left\{\phi_{j}: j=1,2, \ldots\right\}\right]}=\Phi_{X}(\mathcal{B})$ e o Teorema 2.3.4 nos dá que

$$
\overline{\left[\left\{\phi_{j}: j=1,2, \ldots\right\}\right]}=G_{K}(X) .
$$

O resultado segue.

Ainda buscando uma associação entre o operador integral $\mathcal{K}$ e a universalidade do núcleo $\mathrm{PD} K$, temos o próximo resultado, que diz respeito à imagem de $\mathcal{K}$.

Teorema 2.5.2. Se $X$ é um subconjunto compacto e não vazio de $E$, então $G_{K}(X)$ coincide com o fecho da imagem de $\mathcal{K}$ em $C(X)$, isto é, $G_{K}(X)=\overline{\mathcal{K}\left(L^{2}(X, \mu)\right)}$.

Demonstração: Fixado $X$, o Lema 2.2.3 garante que a demonstração resume-se a justificar que

$$
\iota^{-1}\left(\left\{K_{y}: y \in X\right\}^{o}\right)=\iota^{-1}\left(\mathcal{K}\left(L^{2}(X, \mu)\right)^{o}\right) .
$$

Se $\mu \in \iota^{-1}\left(\left\{K_{y}: y \in X\right\}^{o}\right)$, temos imediatamente que

$$
\int_{X} K(x, y) d \mu(x)=0, \quad y \in X .
$$

Por outro lado, se integramos ambos os lados de (2.8) obtemos

$$
\int_{X} \mathcal{K}(g)(x) d \mu(x)=\int_{X} \int_{X} K(x, y) g(y) d \mu(y) d \mu(x), \quad g \in L^{2}(X, \mu) .
$$

Daí, pelo Teorema de Fubini, segue que

$$
\int_{X} \mathcal{K}(g)(x) d \mu(x)=\int_{X} g(y)\left(\int_{X} K(x, y) d \mu(x)\right) d \mu(y)=0, \quad g \in L^{2}(X, \mu) .
$$

Em outras palavras, $\mu \in \iota^{-1}\left(\mathcal{K}\left(L^{2}(X, \mu)\right)\right)$. Reciprocamente, se

$$
\int_{X} \mathcal{K}(g)(x) d \mu(x)=0, \quad g \in L^{2}(X, \mu),
$$

então procedendo como na parte anterior, podemos deduzir que

$$
\int_{X} g(y)\left\{\int_{X} K(x, y) d \mu(x)\right\} d \mu(y)=0, \quad g \in L^{2}(X, \mu) .
$$

Em particular, como a função

$$
g_{\mu}:=\overline{\int_{X} K(x, \cdot) d \mu(x)}
$$

é um elemento de $L^{2}(X, \mu)$, segue que

$$
\int_{X}\left|g_{\mu}(y)\right|^{2} d \mu(y)=\int_{X}\left|\int_{X} K(x, y) d \mu(x)\right|^{2} d \mu(y)=0 .
$$


Da continuidade de $g_{\mu}$ e da igualdade acima, segue que $g_{\mu}$ é identicamente nula. Em outras palavras,

$$
\int_{X} K(x, y) d \mu(x)=0, \quad y \in X
$$

Portanto, $\mu \in \iota^{-1}\left(\left\{K_{y}: y \in X\right\}^{o}\right)$.

O corolário abaixo não necessita justificativa.

Corolário 2.5.3. Seja $X$ um subconjunto compacto e não vazio de E. Então, $G_{K}(X)=C(X)$ se, e somente se, $\overline{\mathcal{K}\left(L^{2}(X, \mu)\right)}=C(X)$.

Finalizamos a seção registrando um resultado de universalidade envolvendo os resultados que acabamos de demonstrar.

Teorema 2.5.4. Seja $\mu$ uma medida sobre $E$ de tal modo que, para cada compacto não vazio $X$ de $E$, a restrição de $\mu$ a $X$ é uma medida de Borel em $X$ e o contexto descrito no começo da seção vale. Então, $K$ é universal se, e somente se, $\overline{\mathcal{K}\left(L^{2}(X, \mu)\right)}=C(X)$ para cada compacto não vazio $X$ de $E$. 



\section{$-\frac{1}{3}$ \\ Exemplificando universalidade}

Neste capítulo analisaremos alguns exemplos concretos, onde os resultados das seções anteriores são aplicáveis. Descreveremos a universalidade do núcleo através de propriedades tão explícitas quanto possíveis.

\subsection{Um núcleo de Mercer clássico}

Neste exemplo, consideraremos um núcleo de Mercer clássico sobre um espaço topológico $E$, ou seja, um núcleo $K: E \times E \rightarrow \mathbb{C}$ da forma

$$
K(x, y):=\sum_{j=1}^{\infty} \phi_{j}(x) \overline{\phi_{j}(y)}, \quad(x, y) \in E \times E,
$$

onde cada função $\phi_{j}$ é contínua em $E$. Assumiremos que a série acima é uniformemente convergente em cada conjunto $X \times X$, onde $X$ é um subconjunto compacto e não vazio de $E$. É imediato verificar que $K$ é positivo definido (e contínuo).

Demonstraremos o seguinte resultado.

Teorema 3.1.1. O núcleo $K$ descrito acima é universal se, e somente se, para cada subconjunto compacto e não vazio $X$ de $E$, o conjunto $\left\{\left.\phi_{j}\right|_{X}: j=1,2, \ldots\right\}$ é fundamental em $C(X)$.

Demonstração: Considerando o espaço de Hilbert $\ell_{2}$ munido de seu produto interno canônico, uma função layout para $K$ é $\Phi: E \rightarrow \ell_{2}$ dada por

$$
\Phi(x)=\left(\phi_{1}(x), \phi_{2}(x), \ldots\right), \quad x \in E .
$$


Assim sendo, usaremos o Teorema 2.4 .3 para justificar o resultado, determinando uma condição para a universalidade de $\Phi$. Fixando a base canônica $\mathcal{B}_{0}$ de $\ell_{2}$, a universalidade de $\Phi$ exige que $\Phi_{X}\left(\mathcal{B}_{0}\right)=C(X)$, qualquer que seja o compacto não vazio $X$ de $E$. É fácil ver que se $v(j)=e^{i}(j) \in \mathcal{B}_{0}$, onde $e^{i}$ é o vetor constituido por um na $i$-ésima entrada e zero nas demais, então

$$
\Phi_{v}(x)=\langle\Phi(x), v\rangle_{\ell_{2}}=\sum_{j=1}^{\infty} \overline{e^{i}(j)} \phi_{j}(x)=\phi_{i}(x), \quad x \in X .
$$

Logo,

$$
\Phi_{X}\left(\mathcal{B}_{0}\right)=\overline{\left[\left\{\Phi_{v}: v \in \mathcal{B}_{0}\right\}\right]}=\overline{\left[\left\{\phi_{j}: j=1,2, \ldots\right\}\right]},
$$

e a universalidade de $\Phi$ corresponde, então, à condição do enunciado.

Observação 3.1.2. O resultado acima continua válido se na representação de $K$ tivermos uma soma finita. Basta trocar o espaço $\ell_{2}$ por um espaço de dimensão finita. Detalhes serão omitidos.

\subsection{Núcleos definidos por séries de potências}

No exemplo desta seção, abordaremos um núcleo positivo definido e contínuo sobre $\mathbb{R}^{d}$ da forma

$$
K(x, y):=\sum_{n=0}^{\infty} a_{n}(x \cdot y)^{n}, \quad x, y \in \mathbb{R}^{d}
$$

onde cada coeficiente $a_{n}$ é positivo. O núcleo $K$ acima é, de fato, PD em $\mathbb{R}^{d}$ por ser um limite pontual de núcleos deste tipo. Por outro lado, as contas abaixo evidenciarão isso diretamente.

No lema abaixo deduziremos uma expressão alternativa para $K$. Empregaremos um pouco de notação multinomial usual. Para um multi-índice $\alpha=\left(\alpha_{1}, \alpha_{2}, \ldots, \alpha_{d}\right) \in \mathbb{Z}_{+}^{d}$, escreveremos

$$
\tilde{\alpha}:=\left(\begin{array}{c}
|\alpha| \\
\alpha
\end{array}\right)=\frac{|\alpha| !}{\alpha_{1} ! \alpha_{2} ! \cdots \alpha_{d} !}
$$

Lema 3.2.1. O núcleo $K$ em (3.1) pode ser escrito na forma

$$
K(x, y)=\sum_{\alpha \in \mathbb{Z}_{+}^{d}} \phi_{\alpha}(x) \phi_{\alpha}(y), \quad x, y \in \mathbb{R}^{d}
$$

onde

$$
\phi_{\alpha}(x):=\left(\tilde{\alpha} a_{|\alpha|}\right)^{1 / 2} x^{\alpha}, \quad \alpha \in \mathbb{Z}_{+}^{d}, \quad x \in \mathbb{R}^{d} .
$$


Demonstração: Escrevendo $x=\left(x_{1}, x_{2}, \ldots, x_{d}\right)$ e $y=\left(y_{1}, y_{2}, \ldots, y_{d}\right)$ e usando o teorema multinomial $([\underline{5}, \mathrm{p} .56])$ em $(x \cdot y)^{n}$ obtemos

$$
K(x, y)=\sum_{n=0}^{\infty} a_{n}\left(\sum_{\alpha_{1}+\alpha_{2}+\ldots+\alpha_{d}=n} \frac{n !}{\alpha_{1} ! \alpha_{2} ! \ldots \alpha_{d} !}\left(x_{1} y_{1}\right)^{\alpha_{1}}\left(x_{2} y_{2}\right)^{\alpha_{2}} \ldots\left(x_{d} y_{d}\right)^{\alpha_{d}}\right), x, y \in \mathbb{R}^{d} .
$$

Um pequeno ajuste transforma a fórmula acima em

$$
K(x, y)=\sum_{n=0}^{\infty}\left(\sum_{|\alpha|=n} a_{|\alpha|} \tilde{\alpha}\left(x_{1} y_{1}\right)^{\alpha_{1}}\left(x_{2} y_{2}\right)^{\alpha_{2}} \ldots\left(x_{d} y_{d}\right)^{\alpha_{d}}\right), \quad x, y \in \mathbb{R}^{d}
$$

ou seja,

$$
K(x, y)=\sum_{\alpha \in \mathbb{Z}_{+}^{d}} a_{|\alpha|} \tilde{\alpha}\left(x_{1} y_{1}\right)^{\alpha_{1}}\left(x_{2} y_{2}\right)^{\alpha_{2}} \ldots\left(x_{d} y_{d}\right)^{\alpha_{d}}, \quad x, y \in \mathbb{R}^{d}
$$

Podemos condensar índices nesta última expressão e chegar em

$$
K(x, y)=\sum_{\alpha \in \mathbb{Z}_{+}^{d}} a_{|\alpha|} \tilde{\alpha} x^{\alpha} y^{\alpha}, \quad x, y \in \mathbb{R}^{d},
$$

e esta é a expressão do enunciado do lema.

Podemos agora enunciar e provar o teorema principal desta seção.

Teorema 3.2.2. O núcleo $K$ em (3.1) é universal.

Demonstração: O lema anterior revela que o núcleo se encaixa na descrição do Teorema 3.1.1 com $E=\mathbb{R}^{d} \mathrm{e}$

$$
\phi_{\alpha}(x):=\left(\tilde{\alpha} a_{|\alpha|}\right)^{1 / 2} x^{\alpha}, \quad \alpha \in \mathbb{Z}_{+}^{d}, \quad x \in \mathbb{R}^{d},
$$

onde agora o conjunto de índices é $\mathbb{Z}_{+}^{d}$ (ainda enumerável). Assim sendo, para provarmos o teorema basta fixarmos um compacto não vazio $X$ de $\mathbb{R}^{d}$ e verificarmos que o conjunto $A:=\left\{\left.\phi_{\alpha}\right|_{X}: \alpha \in \mathbb{Z}_{+}^{d}\right\}$ é fundamental em $C(X)$. Pelo Teorema de StoneWeierstrass $([23,24])$, é suficiente verificarmos que $A$ é uma sub-álgebra que separa pontos em $C(X)$. Tomando-se $\alpha=0$, verificamos que $A$ contém as funções constantes. É relativamente simples verificar que se $f, g \in A$, então $f g \in A$. Ainda, se $r_{1}, r_{2} \in \mathbb{R}$, também temos que $r_{1} f+r_{2} g \in A$. Resta, então, verificarmos que $A$ separa pontos. Sejam $x=\left(x_{1}, x_{2}, \ldots, x_{d}\right)$ e $y=\left(y_{1}, y_{2}, \ldots, y_{d}\right)$ dois pontos distintos de $X$ (se $X$ for unitário, não há nada para ser demonstrado). Existe, então, pelo menos um par de números, $x_{k}$ e $y_{k}$, com $x_{k} \neq y_{k}$. Tomemos o multi-índice $\alpha=\left(\alpha_{1}, \alpha_{2}, \ldots, \alpha_{k}, \ldots, \alpha_{d}\right)$ de $\mathbb{Z}_{+}^{d}$ com $\alpha_{k}=1$ e $\alpha_{j}=0$ para $j \neq k$. Então,

$$
\phi_{\alpha}(x)=x_{k} a_{1}^{1 / 2} \neq y_{k} a_{1}^{1 / 2}=\phi_{\alpha}(y) .
$$


Assim, o Teorema de Stone-Weierstrass implica que o fecho de $A$ em $C(X)$ é o próprio $C(X)$. Isto completa a prova.

O teorema anterior pode ser generalizado para o contexto complexo. Para isto, o núcleo $K: \mathbb{C}^{d} \times \mathbb{C}^{d} \rightarrow \mathbb{C}$ tem que ser da forma

$$
K(z, w):=\sum_{m, n=0}^{\infty} a_{m, n}(z \cdot w)^{m}(\overline{z \cdot w})^{n}, \quad z, w \in \mathbb{C}^{d},
$$

onde "." é o produto escalar usual em $\mathbb{C}^{d}$ e os coeficientes $a_{m, n}$ são todos positivos. A representação para $K$ do lema toma a forma

$$
K(z, w)=\sum_{\alpha, \beta \in \mathbb{Z}_{+}^{d}} \phi_{\alpha, \beta}(z) \overline{\phi_{\alpha, \beta}(w)}, \quad z, w \in \mathbb{C}^{d},
$$

onde

$$
\phi_{\alpha, \beta}(z):=\left(\tilde{\alpha} \tilde{\beta} a_{|\alpha|,|\beta|}\right)^{1 / 2} z^{\alpha} \bar{z}^{\beta}, \quad \alpha, \beta \in \mathbb{Z}_{+}^{d}, \quad z \in \mathbb{R}^{d} .
$$

Na demonstração da versão complexa do Teorema 3.2 .2 é necessário utilizar a versão complexa do teorema de Stone-Weierstrass. Os detalhes serão omitidos.

\subsection{Núcleos PD sobre a esfera unitária}

Nesta seção consideraremos o núcleo contínuo e PD $K$ sobre a esfera unitária $S^{d}$ introduzido no Exemplo 1.1.4:

$$
K(x, y)=\sum_{k=0}^{\infty} a_{k} P_{k}^{d}(x \cdot y), \quad x, y \in \mathbb{S}^{d} .
$$

O resultado que descreve uma equivalência para a universalidade de $K$ é como segue.

Teorema 3.3.1. O núcleo $K$ acima é universal se, e somente se, cada coeficiente $a_{k}$ é positivo.

Demonstração: Primeiramente há de se notar que na verificação da definição de universalidade neste caso, basta considerar o caso $X=S^{d}$, uma vez que a própria $S^{d}$ já é compacta. Como no exemplo anterior, a ideia aqui é reescrever a expressão que define $K$, colocando-a no formato de um núcleo PD clássico. Para tanto, seja $\left\{Y_{k j}: j=1,2, \ldots, N(k, d)\right\}$ uma base ortonormal (com relação ao produto interno de $L^{2}\left(S^{d}\right)$ usual) do espaço de todos os harmônicos esféricos de grau $k$ em $d+1$ variáveis. O teorema da adição para harmônicos esféricos ([12]) nos informa que para cada $k$,

$$
P_{k}^{d}(x \cdot y)=\frac{\sigma_{d}}{N(k, d)} \sum_{j=1}^{N(k, d)} Y_{k j}(x) \overline{Y_{k j}(y)}, \quad x, y \in S^{d},
$$


onde $\sigma_{d}$ é a área de superfície de $S^{d}$. Logo, a representação de $K$ toma a forma

$$
K(x, y)=\sigma_{d} \sum_{k=0}^{\infty} \frac{a_{k}}{N(k, d)} \sum_{j=1}^{N(k, d)} \overline{Y_{k j}(x) Y_{k j}(y)}, \quad x, y \in S^{d} .
$$

Em outras palavras, definindo $\mathcal{I}:=\left\{(k, l): k \in \mathbb{Z}_{+}, l=1,2, \ldots, N(k, d)\right\}$, temos que

$$
K(x, y)=\sum_{(k, l) \in \mathcal{I}} \phi_{k l}(x) \overline{\phi_{k l}(y)}, \quad x, y \in S^{d}
$$

onde

$$
\phi_{k l}(x):=\sigma_{d}^{1 / 2}\left(\frac{a_{k}}{N(k, d)}\right)^{1 / 2} Y_{k l}(x), \quad x \in S^{d}, \quad(k, l) \in \mathcal{I} .
$$

Um resultado clássico da análise na esfera garante que $\left\{\phi_{k l}:(k, l) \in \mathcal{I}\right\}$ é fundamental em $C\left(S^{d}\right)$, enquanto que a fundamentalidade se perde quando qualquer par $(k, l)$ é omitido na indexação do conjunto. Assim, lembrando o Teorema 3.1.1, deduzimos que $K$ é universal se, e somente se, cada coeficiente $a_{k}$ é positivo.

Observamos que existe uma versão similar do teorema acima para núcleos PD sobre a esfera unitária de $\mathbb{C}^{q}$. Não incluiremos os detalhes aqui, preferindo analisar casos que não tem tanta semelhança com o anterior.

\subsection{Núcleos invariantes por translação sobre $\mathbb{R}^{d}$}

Nesta seção consideraremos núcleos $K: \mathbb{R}^{d} \times \mathbb{R}^{d} \rightarrow \mathbb{C}$ da forma

$$
K(x, y)=\kappa(x-y), \quad x, y \in \mathbb{R}^{d},
$$

onde $\kappa: \mathbb{R}^{d} \rightarrow \mathbb{C}$ é uma função contínua conveniente. Núcleos deste tipo não se alteram quando ambas as variáveis $x$ e $y$ são transladadas por um mesmo vetor de $\mathbb{R}^{d}$.

O seguinte resultado clássico de Bochner ([6]) descreve com exatidão quais funções $\kappa$ produzem um núcleo $K \mathrm{PD}$ sobre $\mathbb{R}^{d}$ via 3.2 .

Proposição 3.4.1. A aplicação $K$ definida em (3.2) é um núcleo PD e contínuo se, e somente se, existe uma única medida de Borel finita e não negativa $\mu$ sobre $\mathbb{R}^{d}$ tal que

$$
\kappa(x)=\int_{\mathbb{R}^{d}} e^{i(x \cdot y)} d \mu(y), \quad x \in \mathbb{R}^{d} .
$$

Se um núcleo $K$ como em 3.2 é $\mathrm{PD}$ sobre $\mathbb{R}^{d}$, escreveremos $K=K_{\mu}$ para indicar qual é a medida $\mu$ da proposição que está atrelada à representação da função $\kappa$ correspondente. O objetivo principal nesta seção é então descrever a universalidade de $K_{\mu}$ através de alguma propriedade envolvendo a medida $\mu$. 
No primeiro resultado descreveremos a função layout para $K$. Lembramos que o suporte $\operatorname{supp}(\mu)$ de uma medida de Borel $\mu$ é o conjunto de todos os pontos $x$ de $\mathbb{R}^{d}$ para os quais qualquer bola aberta de $\mathbb{R}^{d}$ centrada em $x$ tem medida positiva. Em outras palavras,

$$
\operatorname{supp}(\mu)=\mathbb{R}^{d} \backslash \cup\left\{\mathcal{O}: \mathcal{O} \text { é aberto em } \mathbb{R}^{d} \text { e } \mu(\mathcal{O})=0\right\}
$$

O suporte de $\mu$ é um subconjunto fechado de $\mathbb{R}^{d}$ e $\mu\left(\mathbb{R}^{d} \backslash \operatorname{supp}(\mu)\right)=0$. Este conceito e as propriedades listadas acima são discutidos em [13, p.122] e também em [, p.88].

Proposição 3.4.2. Seja $\mu$ uma medida de Borel finita e não negativa sobre $\mathbb{R}^{d}$. Se $\mathcal{W}$ é o espaço de Hilbert $L^{2}(\operatorname{supp}(\mu), \mu)$ usual, então uma função layout para $K_{\mu}$ é $\Phi: \mathbb{R}^{d} \rightarrow \mathcal{W}$ dada por

$$
\Phi(x)(y):=e^{i(x \cdot y)}, \quad x \in \mathbb{R}^{d}, \quad y \in \operatorname{supp}(\mu) .
$$

Demonstração: Escrevamos $\langle\cdot, \cdot\rangle_{\mathcal{W}}$ para indicar o produto interno de $\mathcal{W}$ definido por

$$
\langle f, g\rangle_{\mathcal{W}}:=\int_{\operatorname{supp}(\mu)} f(x) \overline{g(x)} d \mu(x), \quad f, g \in \mathcal{W} .
$$

Como $\mu\left(\mathbb{R}^{d} \backslash \operatorname{supp}(\mu)\right)=0$,

$$
\begin{aligned}
\langle\Phi(x), \Phi(y)\rangle_{\mathcal{W}} & =\int_{\operatorname{supp}(\mu)} e^{i[(x-y) \cdot w]} d \mu(w) \\
& =\int_{\operatorname{supp}(\mu)} e^{i[(x-y) \cdot w]} d \mu(w)+\int_{\mathbb{R}^{d} \backslash \operatorname{supp}(\mu)} e^{i[(x-y) \cdot w]} d \mu(w), \quad x, y \in \mathbb{R}^{d} .
\end{aligned}
$$

Logo,

$$
\langle\Phi(x), \Phi(y)\rangle_{\mathcal{W}}=\int_{\mathbb{R}^{d}} e^{i[(x-y) \cdot w]} d \mu(w)=\kappa(x-y)=K_{\mu}(x, y), \quad x, y \in \mathbb{R}^{d},
$$

como queríamos.

Antes de prosseguir, notemos que a imagem de $\Phi$ contém tão somente funções contínuas.

Lema 3.4.3. Sejam $\mu$ uma medida de Borel finita e não negativa sobre $\mathbb{R}^{d}$ e $\Phi$ a função layout para $K_{\mu}$ definida na proposição anterior. Se $X$ é um subconjunto compacto $e$ não vazio de $\mathbb{R}^{d}$, então ker $j_{X}$ coincide com a imagem inversa por $\iota$ do anulador do conjunto

$$
\exp (\mu):=\{\Phi(x) \in C(X): x \in \operatorname{supp}(\mu)\}
$$

em $C(X)$, ou seja, ker $j_{X}=\iota^{-1}\left(\exp (\mu)^{o}\right)$. 
Demonstração: Seja $X$ um subconjunto compacto e não vazio de $\mathbb{R}^{d}$. Se $\nu \in \mathcal{M}(X)$ é tal que $j_{X}(\nu)=0$, então

$$
\begin{aligned}
0=\left\langle j_{X}(\nu), f\right\rangle_{\mathcal{W}} & =\int_{X}\langle\Phi(x), f\rangle_{\mathcal{W}} d \nu(x) \\
& =\int_{X} \int_{\operatorname{supp}(\mu)} \Phi(x)(w) \overline{f(w)} d \mu(w) d \nu(x), \quad f \in \mathcal{W} .
\end{aligned}
$$

Embutindo a definição de $\Phi$ e trocando a ordem de integração vem que

$$
0=\int_{\operatorname{supp}(\mu)}\left(\int_{X} e^{i(x \cdot w)} d \nu(x)\right) \overline{f(w)} d \mu(w), \quad f \in L^{2}(\operatorname{supp}(\mu), \mu) .
$$

Logo, a função $h \in L^{2}(\operatorname{supp}(\mu), \mu)$ dada por

$$
h(w)=\int_{X} e^{i(x \cdot w)} d \nu(x), \quad w \in \operatorname{supp}(\mu)
$$

é ortogonal a cada elemento de $L^{2}(\operatorname{supp}(\mu), \mu)$. Como $L^{2}(\operatorname{supp}(\mu), \mu)$ é um espaço de Hilbert, segue que $h$ é identicamente nula em $L^{2}(\operatorname{supp}(\mu), \mu)$, ou seja, $\iota(\nu) \in \exp (\mu)^{o}$. Claramente, estes argumentos podem ser revertidos para justificar a outra inclusão.

Corolário 3.4.4. Sejam $\mu$ e $\Phi$ como descritos na proposição anterior. Se $X$ é um subconjunto compacto não vazio de $\mathbb{R}^{d}$, então $G_{K}(X)=C(X)$ se, e somente se, $\exp (\mu)$ é fundamental em $C(X)$.

Demonstração: Seja $X$ um subconjunto compacto e não vazio de $\mathbb{R}^{d}$. Lembrando o Lema 2.2 .5 e usando o resultado anterior, podemos deduzir que $j_{X}$ é injetora se, e somente se, $\exp (\mu)$ é fundamental em $C(X)$. Por outro lado, o Teorema 2.2.6 revela que $j_{X}$ é injetora se, e somente se, $G_{K_{\mu}}(X)=C(X)$.

Desta forma, temos a seguinte equivalência para a universalidade de $K_{\mu}$.

Teorema 3.4.5. Seja $\mu$ uma medida de Borel finita e não negativa sobre $\mathbb{R}^{d}$. O núcleo $K_{\mu}$ é universal se, e somente se, $\overline{[\exp (\mu)]}=C(X)$, qualquer que seja o subconjunto compacto e não vazio $X$ de $\mathbb{R}^{d}$.

O resultado a seguir permite obtermos a universalidade de um núcleo a partir da universalidade de outro.

Corolário 3.4.6. Sejam $\mu$ e $\nu$ medidas de Borel finitas e não negativas sobre $\mathbb{R}^{d}$. Se $\operatorname{supp}(\mu)=\operatorname{supp}(\nu)$, então o núcleo $K_{\mu}$ é universal se, e somente se, $K_{\nu}$ é universal.

Demonstração: É suficiente observar que para dois núcleos $K_{\mu}$ e $K_{\nu}$ com $\operatorname{supp}(\mu)=\operatorname{supp}(\nu)$, temos necessariamente que $\exp (\mu)=\exp (\nu)$ e, em seguida, usar o teorema anterior. 
No próximo resultado descreveremos uma condição suficiente para a universalidade de $K_{\mu}$. A condição faz uso de um conceito adicional que passamos a descrever. Um subconjunto $A$ de $\mathbb{C}^{d}$ é um conjunto de unicidade para $\mathbb{C}^{d}$ se a única função inteira que se anula em todos os pontos de $A$ é a função nula.

Proposição 3.4.7. Seja $\mu$ uma medida de Borel finita e não negativa sobre $\mathbb{R}^{d}$. Se supp $(\mu)$ é um conjunto de unicidade para $\mathbb{C}^{d}$, então $K_{\mu}$ é universal.

Demonstração: Provaremos que se $\operatorname{supp}(\mu)$ é um conjunto de unicidade para $\mathbb{C}^{d}$, então $j_{X}$ é injetora, qualquer que seja subconjunto compacto e não vazio $X$ de $\mathbb{R}^{d}$. Fixe $X$ e suponha que exista uma medida não nula $\nu$ em $\mathcal{M}(X)$ tal que $j_{X}(\nu)=0$. Consultando o Lema 3.4.3 e sua demonstração, concluímos que

$$
\int_{X} e^{i(x \cdot y)} d \nu(x)=0, \quad y \in \operatorname{supp}(\mu)
$$

Em outras palavras, a função inteira $g$ dada por

$$
g(z):=\int_{X} e^{i(x \cdot z)} d \nu(x), \quad z \in \mathbb{C}^{d}
$$

anula-se em $\operatorname{supp}(\nu)$. Se este é um conjunto de unicidade para $\mathbb{C}^{d}$, poderemos concluir que $g$ é identicamente nula em $\mathbb{C}^{d}$. Calculando $g$ em pontos da forma $-i y, y \in \mathbb{R}^{d}$, obtemos

$$
\int_{X} e^{x \cdot y} d \nu(x)=0, \quad y \in \mathbb{R}^{d}
$$

Como o integrando é positivo, isso só ocorre se $\nu=0$. O Teorema 2.2.6 nos garante o resultado.

Corolário 3.4.8. Seja $\mu$ uma medida de Borel finita e não negativa sobre $\mathbb{R}^{d}$. Se $\operatorname{supp}(\mu)$ tem medida de Lebesgue não negativa em $\mathbb{R}^{d}$, então $K_{\mu}$ é universal.

Demonstração: Um subconjunto de $\mathbb{R}^{d}$ com medida de Lebesgue não negativa é necessariamente um conjunto de unicidade para $\mathbb{C}^{d}$. Isso é consequência do seguinte fato: o conjunto dos zeros reais de uma função inteira em $\mathbb{C}^{d}$ é um conjunto com medida de Lebesgue nula em $\mathbb{R}^{d}([14$, p.102]). Assim, o resultado segue da proposição anterior.

A seguir, apresentaremos outra crondição para a universalidade de $K_{\mu}$, agora usando o Teorema da Decomposição de Lebesgue ([18, p.278]). Denote por $m$ a medida de Lebesgue usual sobre $\mathbb{R}^{d}$. Se $\nu$ é uma medida de Borel finita não negativa sobre $\mathbb{R}^{d}$, tal teorema garante que $\nu$ pode ser decomposta de maneira única na forma $\nu=\nu_{c}+\nu_{s}$, onde $\nu_{c}$ é uma medida não negativa absolutamente contínua em relação a $m$, isto 
é, $\nu_{c}(A)=0$ sempre que $m(A)=0$, enquanto que $\nu_{s}$ é uma medida não negativa mutualmente singular a $m$, isto é, existem $A, B \subseteq \mathbb{R}^{d}$ disjuntos tais que $\mathbb{R}^{d}=A \cup B$ e $m(X)=\nu_{s}(Y)=0$, quaisquer que sejam $X$ em $A$ e $Y$ em $B$.

O contexto acima ainda permite a aplicação do Teorema de Radon-Nikodym (18, p.276]) para $\nu_{c}$. Consequentemente, existe uma função não negativa $g_{\nu} \mathrm{em} L^{1}\left(\mathbb{R}^{d}\right)$ de modo que

$$
\nu_{c}(A)=\int_{A} g_{\nu}(x) d m(x),
$$

qualquer que seja o subconjunto de Borel $A$ de $\mathbb{R}^{d}$.

Concluída esta pequena revisão do Teorema da Decomposição de Lebesgue, podemos enunciar e demonstrar mais uma condição para a universalidade para um núcleo $K_{\mu}$. Na demonstração, utilizaremos a seguinte definição do suporte de uma função $f$ de $L^{1}\left(\mathbb{R}^{d}, m\right)([7, \mathrm{p} .105])$

$$
\operatorname{supp}(f)=\bigcap\left\{F \subset \mathbb{R}^{d}: F \text { é fechado em } \mathbb{R}^{d} \text { e } f=0, m \text {-q.s. em } \mathbb{R}^{d} \backslash F\right\} .
$$

Proposição 3.4.9. Seja $\mu$ uma medida de Borel finita e não negativa sobre $\mathbb{R}^{d}$. Se na decomposição de Lebesgue de $\mu$, a medida $\mu_{c}$ é não nula sobre os conjuntos $m$ mensuráveis, então $K_{\mu}$ é universal.

Demonstração: A ideia da demonstração é deduzir que, na condição de $\mu_{c}$ ser não nula, $\operatorname{supp}(\mu)$ tem medida de Lebesgue não negativa e, então, aplicar o corolário anterior. Mostremos inicialmente que $\operatorname{supp}\left(g_{\mu}\right) \subseteq \operatorname{supp}(\mu)$. Para tanto, considere os conjuntos $C_{g_{\mu}}=\left\{F \subset \mathbb{R}^{d}: F\right.$ é fechado em $\mathbb{R}^{d}$ e $g_{\mu}=0 m$-q.s. em $\left.\mathbb{R}^{d} \backslash F\right\}$ e $C_{\mu}=\left\{F \subset \mathbb{R}^{d}: F\right.$ é fechado em $\mathbb{R}^{d}$ e $\left.\mu\left(\mathbb{R}^{d} \backslash F^{\prime}\right)=0\right\}$. Seja $F^{\prime}$ um elemento arbitrário de $C_{\mu}$. A equação (3.4) nos mostra que

$$
\int_{\mathbb{R}^{d} \backslash F^{\prime}} g_{\mu}(x) d m(x)=\mu_{c}\left(\mathbb{R}^{d} \backslash F^{\prime}\right),
$$

e pela decomposição de Lebesgue obtemos

$$
\int_{\mathbb{R}^{d} \backslash F^{\prime}} g_{\mu}(x) d m(x)=\mu\left(\mathbb{R}^{d} \backslash F^{\prime}\right)-\mu_{s}\left(\mathbb{R}^{d} \backslash F^{\prime}\right) .
$$

Da hipótese de $\mathbb{R}^{d} \backslash F^{\prime}$ ser um conjunto de medida $\mu$ nula, segue que

$$
\int_{\mathbb{R}^{d} \backslash F^{\prime}} g_{\mu}(x) d m(x)=-\mu_{s}\left(\mathbb{R}^{d} \backslash F^{\prime}\right) .
$$

Uma vez que $\mu_{s}$ é mutualmente singular a medida de Lebesgue $m$, existem $A, B \subseteq \mathbb{R}^{d}$ disjuntos tais que $\mathbb{R}^{d}=A \cup B$ e, para quaiquer $X \subseteq A$ e $Y \subseteq B$, tem-se $m(X)=$ $\mu_{s}(Y)=0$. Com tais conjuntos reescrevemos

$$
\int_{\mathbb{R}^{d} \backslash F^{\prime}} g_{\mu}(x) d m(x)=-\mu_{s}\left((A \cup B) \backslash F^{\prime}\right)=-\left(\mu_{s}\left(A \backslash F^{\prime}\right)+\mu_{s}\left(B \backslash F^{\prime}\right)\right) .
$$


É fácil ver que $\mu_{s}\left(B \backslash F^{\prime}\right)=0$, logo a fórmula acima se torna

$$
\int_{\mathbb{R}^{d} \backslash F^{\prime}} g_{\mu}(x) d m(x)=-\mu_{s}\left(A \backslash F^{\prime}\right)
$$

e usando novamente a expressão fornecida pela decomposição de Lebesgue

$$
\int_{\mathbb{R}^{d} \backslash F^{\prime}} g_{\mu}(x) d m(x)=-\mu\left(A \backslash F^{\prime}\right)+\mu_{c}\left(A \backslash F^{\prime}\right) .
$$

Novamente da hipótese de $\mu\left(\mathbb{R}^{d} \backslash F^{\prime}\right)=0$, juntamente ao fato de $\mu$ ser de Borel, temos que $\mu\left(A \backslash F^{\prime}\right)=0$. Logo,

$$
\int_{\mathbb{R}^{d} \backslash F^{\prime}} g_{\mu}(x) d m(x)=\mu_{c}\left(A \backslash F^{\prime}\right),
$$

e pela fórmula do teorema de Radon-Nikodym,

$$
\int_{\mathbb{R}^{d} \backslash F^{\prime}} g_{\mu}(x) d m(x)=\int_{A \backslash F^{\prime}} g_{\mu}(x) d m(x) .
$$

Como $m(A)=0$, segue trivialmente que $m\left(A \backslash F^{\prime}\right)=0$. Com isso a integral do lado direito da última equação é nula e consequentemente o mesmo ocorre com o termo do lado esquerdo. Logo $g_{\mu}=0 m$-q.s em $\mathbb{R}^{d} \backslash F^{\prime}$ e, consequentemente, $F^{\prime} \in C_{g_{\mu}}$. Portanto $C_{\mu} \subseteq C_{g \mu}$ e então

$$
\bigcap_{F \in C_{g_{\mu}}} F \subseteq \bigcap_{F \in C_{\mu}} F
$$

Assim, pelas fórmulas (3.3) e (3.5), obtemos a inclusão desejada. Por fim, da igualdade (3.4) obtemos trivialmente que se $\mu_{c}$ é não nula então $g_{\mu}$ não pode ser identicamente nula. Mais do que isso, tal equação mostra que $m\left(\operatorname{supp} g_{\mu}\right)>0$. Assim, existe um conjunto de Borel $M$ tal que $M \subseteq \operatorname{supp} g_{\mu}$ e $m(M)>0$, e a inclusão $\operatorname{supp} g_{\mu} \subseteq \operatorname{supp}(\mu)$ nos garante o resultado.

\subsection{Núcleos radiais sobre $\mathbb{R}^{d}$}

Nesta seção, consideraremos os núcleos positivos definidos sobre $\mathbb{R}^{d}$ que são radiais. Um núcleo contínuo $K$ sobre $\mathbb{R}^{d}$ é radial se existir uma função contínua $g: \mathbb{R}_{+} \rightarrow \mathbb{R}$ satisfazendo

$$
K(x, y):=g\left(\|x-y\|^{2}\right), \quad x, y \in \mathbb{R}^{d}
$$

onde $\|\cdot\|$ indica a norma euclidiana do espaço $\mathbb{R}^{d}$. É fácil notar que um núcleo radial é necessariamente invariante por translações das variáveis envolvidas. Em outras palavras, estaremos estudando aqui uma subcategoria especial dos núcleos considerados na seção anterior. 
Os núcleos contínuos, PD e radiais foram caracterizados em [20]. Em tal referência encontra-se a demonstração do resultado que segue.

Proposição 3.5.1. Seja $K$ um núcleo contínuo e PD sobre $\mathbb{R}^{d}$. Então, $K$ é radial se, e somente se, a função $g$ em (3.6) é da forma

$$
g(t)=\int_{0}^{\infty} e^{-t r} d \tau(r), \quad t \in[0, \infty)
$$

onde $\tau$ é uma medida de Borel finita e não negativa em $[0, \infty)$.

Discutiremos agora a universalidade de um núcleo como acima.

Teorema 3.5.2. Seja $K$ um núcleo contínuo, $P D$ e radial sobre $\mathbb{R}^{d}$. Então, $K$ é universal se, e somente se, a medida $\tau$ descrita em (3.7) não é concentrada em $r=0$.

Demonstração: Se $\tau$ é concentrada em $r=0$, então $K$ é necessariamente constante e, portanto, não é universal. Reciprocamente, suponhamos que $\tau$ não está concentrada em 0. Note, então, que supp $(\tau)$ contém pelo menos um número real positivo. Comecemos observando que a representação para $K$ é

$$
K(x, y)=\int_{0}^{\infty} e^{-r\|x-y\|^{2}} d \tau(r), \quad x, y \in \mathbb{R}^{d} .
$$

Calculando a norma na expressão acima e reagrupando, chegamos na expressão

$$
K(x, y)=\int_{0}^{\infty} e^{-r\left(\|x\|^{2}+\|y\|^{2}\right)} e^{2 r(x \cdot y)} d \tau(r), \quad x, y \in \mathbb{R}^{d} .
$$

Usando a série de Maclaurin para a função exponencial usual e também o teorema multinomial, podemos reescrever a expressão acima na forma

$$
K(x, y)=\int_{0}^{\infty} e^{-r\left(\|x\|^{2}+\|y\|^{2}\right)}\left(\sum_{\alpha \in \mathbb{Z}_{+}^{d}} \frac{2^{|\alpha|}}{\alpha_{1} ! \alpha_{2} ! \cdots \alpha_{d} !} r^{|\alpha|} x^{\alpha} y^{\alpha}\right) d \tau(r), \quad x, y \in \mathbb{R}^{d} .
$$

Como $\tau\left(\mathbb{R}_{+} \backslash \operatorname{supp}(\tau)\right)=0$, a expressão acima reduz-se a

$$
K(x, y)=\sum_{\alpha \in \mathbb{Z}_{+}^{d}} \frac{2^{|\alpha|}}{\alpha_{1} ! \alpha_{2} ! \cdots \alpha_{d} !} \int_{\operatorname{Supp}(\tau)} r^{|\alpha|} x^{\alpha} e^{-r\|x\|^{2}} y^{\alpha} e^{-r\|y\|^{2}} d \tau(r) .
$$

Esta representação para $K$ sugere considerarmos o espaço de funções

$$
\mathcal{W}_{\tau}=\left\{F: \mathbb{Z}_{+}^{d} \times \operatorname{supp}(\tau) \rightarrow \mathbb{R}: F(\alpha, \cdot) \in L^{2}(\operatorname{supp}(\tau), \tau), \alpha \in \mathbb{Z}_{+}^{d}\right\},
$$

munido do produto interno

$$
\langle F, G\rangle_{\tau}:=\sum_{\alpha \in \mathbb{Z}_{+}^{d}} \frac{2^{|\alpha|}}{\alpha_{1} ! \alpha_{2} ! \cdots \alpha_{d} !} \int_{\operatorname{supp}(\tau)} F(\alpha, r) G(\alpha, r) d \tau(r), \quad F, G \in \mathcal{W}_{\tau}
$$


É fácil ver que este espaço é de Hilbert. Se $\Phi: \mathbb{R}^{d} \rightarrow \mathcal{W}_{\tau}$ é dada por

$$
\Phi(x)(\alpha, r):=r^{|\alpha| / 2} x^{\alpha} e^{-r\|x\|^{2}}, \quad x \in \mathbb{R}^{d},
$$

então $\Phi$ está bem definida, $\Phi(x) \in \mathcal{W}_{\tau}, x \in \mathbb{R}^{d} \mathrm{e}$

$$
K(x, y)=\langle\Phi(x), \Phi(y)\rangle_{\mathcal{W}_{\tau}}, \quad x, y \in \mathbb{R}^{d}
$$

ou seja, $\Phi$ é uma função layout para $K$. Assim sendo, fixaremos um subconjunto compacto não vazio $X$ de $\mathbb{R}^{d}$ e analisaremos a injetividade da função $j_{X}$ introduzida na Seção 2.1. Se $\mu \in \mathcal{M}(X)$ e $F \in \mathcal{W}_{\tau}$, a equação (2.3) toma a forma

$$
\begin{aligned}
\left\langle j_{X}(\mu), F\right\rangle_{\tau} & =\int_{X}\left(\sum_{\alpha \in \mathbb{Z}_{+}^{d}} \frac{2^{|\alpha|}}{\alpha_{1} ! \alpha_{2} ! \cdots \alpha_{d} !} \int_{\operatorname{Supp}(\tau)} \Phi(x)(\alpha, r) F(\alpha, r) d \tau(r)\right) d \mu(x) \\
& =\int_{X}\left(\sum_{\alpha \in \mathbb{Z}_{+}^{d}} \frac{2^{|\alpha|}}{\alpha_{1} ! \alpha_{2} ! \cdots \alpha_{d} !} \int_{\operatorname{Supp}(\tau)} r^{|\alpha| / 2} x^{\alpha} e^{-r\|x\|^{2}} F(\alpha, r) d \tau(r)\right) d \mu(x) .
\end{aligned}
$$

Trocando a série com a integral vem que

$$
\left\langle j_{X}(\mu), F\right\rangle_{\tau}=\sum_{\alpha \in \mathbb{Z}_{+}^{d}} \frac{2^{|\alpha|}}{\alpha_{1} ! \alpha_{2} ! \cdots \alpha_{d} !} \int_{X}\left(\int_{\operatorname{Supp}(\tau)} r^{|\alpha| / 2} x^{\alpha} e^{-r\|x\|^{2}} F(\alpha, r) d \tau(r)\right) d \mu(x),
$$

enquanto que uma troca na ordem de integração permite reescrevermos a igualdade acima na forma

$$
\left\langle j_{X}(\mu), F\right\rangle_{\tau}=\sum_{\alpha \in \mathbb{Z}_{+}^{d}} \frac{2^{|\alpha|}}{\alpha_{1} ! \alpha_{2} ! \cdots \alpha_{d} !} \int_{\operatorname{supp}(\tau)} F(\alpha, r)\left(\int_{X} r^{|\alpha| / 2} x^{\alpha} e^{-r\|x\|^{2}} d \mu(x)\right) d \tau(r) .
$$

Se $j_{X}(\mu)=0$, esta última igualdade revela que a função

$$
(\alpha, r) \in \mathbb{Z}_{+}^{d} \times \operatorname{supp}(\tau) \rightarrow \int_{X} r^{|\alpha| / 2} x^{\alpha} e^{-r\|x\|^{2}} d \mu(x)
$$

é ortogonal a cada função de $\mathcal{W}_{\tau}$. Como este é um espaço de Hilbert, segue que tal função é identicamente nula em $\mathcal{W}_{\tau}$. Portanto, como o suporte de $\tau$ contém algum $r_{0}>0$, podemos concluir que

$$
\int_{X} r_{0}^{|\alpha| / 2} x^{\alpha} e^{-r_{0}\|x\|^{2}} d \mu(x)=0, \quad \alpha \in \mathbb{Z}_{+}^{d}
$$

ou seja,

$$
\int_{X} x^{\alpha} e^{-r_{0}\|x\|^{2}} d \mu(x)=0, \quad \alpha \in \mathbb{Z}_{+}^{d}
$$

Como o conjunto $\left\{x^{\alpha}: \alpha \in \mathbb{Z}_{+}^{d}\right\}$ é denso em $C(X)$ e a função exponencial no integrando acima é estritamente positiva, o Teorema de Hahn-Banach implica que $\mu=0$. Assim, a função $j_{X}$ é injetora e o Teorema 2.2.6 nos dá que $G_{K}(X)=C(X)$. O resultado descrito no enunciado segue. 
Finalizemos a seção apresentando um exemplo simples para ilustrar o último teorema. Considere o núcleo

$$
K(x, y):=e^{-\alpha\|x-y\|^{2}}, \quad x, y \in \mathbb{R}^{d}, \quad \alpha \in \mathbb{R}_{+} .
$$

Se $\tau$ é a medida de Borel em $\mathbb{R}^{d}$ definida por $\tau(\alpha)=1$ e $\tau(x)=0, x \neq \alpha$, então estamos nas hipóteses da Proposição 3.5.1 garantindo que $K$ é um núcleo radial. Além disso, o Teorema 3.5.2 nos garante que tal núcleo é universal. 

Capítulo

4

\section{Ortogonalidade a partir de suportes disjuntos}

Neste capítulo, consideraremos núcleos contínuos e positivos definidos sobre um espaço topológico $E$. Fixada uma função layout $\Phi: E \rightarrow \mathcal{W}$, analisaremos a seguinte propriedade em $\mathcal{H}_{K}$ : sob quais condições duas funções de $\mathcal{H}_{K}$ são sempre ortogonais, quando seus suportes são subconjuntos disjuntos de $E$ ? Tanto quanto possível, nossos resultados envolverão predominantemente a função layout $\Phi$.

\subsection{Conceitos preliminares e um exemplo}

Neste capítulo, todas as funções que consideraremos serão contínuas a valores complexos. No caso de uma função de $C(E)$, a definição de suporte que introduzimos em (3.5) retorna ao seu formato mais comum, ou seja, $\operatorname{supp}(f)$ é o fecho do conjunto $\{x \in E: f(x) \neq 0\}$ em $E$.

Definição 4.1.1. Seja $\left(\mathcal{H},\langle\cdot, \cdot\rangle_{\mathcal{H}}\right)$ um espaço de Hilbert inteiramente formado por funções contínuas em E. Diremos que $\mathcal{H}$ possui ortogonalidade a partir de suportes disjuntos se quaisquer duas funções $f$ e $g$ de $\mathcal{H}$ com suportes disjuntos são ortogonais em $\mathcal{H}$. Em símbolos, se supp $(f) \cap \operatorname{supp}(g)=\emptyset$, então $\langle f, g\rangle_{\mathcal{H}}=0$.

À primeira vista, podemos ter a impressão de que qualquer espaço de Hilbert de funções contínuas terá a propriedade da ortogonalidade a partir de suportes disjuntos. Nosso primeiro objetivo será, então, o de exibir um exemplo de espaço de Hilbert que não tem tal propriedade. 
Exemplo 4.1.2. Sejam $E$ um espaço topológico discreto ( $E$ é um conjunto finito munido da topologia discreta) e $\mathcal{H}$ o espaço das funções a valores complexos definidas em $E$. Sem perda de generalidade, escreveremos $E=\{1,2, \ldots, d\}$, para algum natural $d$. Como todos os subconjuntos de $E$ são abertos, qualquer função complexa com domínio $E$ é contínua. Podemos ainda identificar naturalmente uma tal função $f$ com sua imagem através da fórmula

$$
f:=(f(1), f(2), \ldots, f(d))
$$

Fixada uma matriz $A$ positiva definida de ordem $d$, a forma quadrática

$$
\langle f, g\rangle_{\mathcal{H}}:=\sum_{i=1}^{d} \sum_{j=1}^{d} f(i) \overline{g(j)} A_{j i}, \quad f, g \in \mathcal{H},
$$

faz com que $\left(\mathcal{H},\langle\cdot, \cdot\rangle_{\mathcal{H}}\right)$ seja um espaço de Hilbert. Neste contexto, se $f \in \mathcal{H}$, então $\operatorname{supp}(f)=\{j: f(j) \neq 0\}$. Logo, se $f, g \in \mathcal{H}$ e $\operatorname{supp}(f) \cap \operatorname{supp}(g)=\emptyset$, então para cada $j \in\{1,2, \ldots, d\}, f(j)$ e $g(j)$ não são simultaneamente não nulos e, consequentemente,

$$
\langle f, g\rangle_{\mathcal{H}}=\sum_{i=1}^{d} \sum_{j=1}^{d} f(i) \overline{g(j)} A_{j i}=\sum_{i \neq j}^{d} f(i) \overline{g(j)} A_{j i} .
$$

Desta forma, se $A$ é diagonal, o espaço tem ortogonalidade a partir de suportes disjuntos. Se $A$ não é diagonal e $A_{p q} \neq 0$ para dois índices distintos $p$ e $q$, podemos definir $f$ e $g$ em $\mathcal{H}$ satisfazendo $f(p)=g(q)=1$ e $f(i)=g(j)=0,1 \leq i, j \leq d, i \neq p$ e $j \neq q$, para obter $f$ e $g$ com suportes disjuntos e $\langle f, g\rangle_{\mathcal{H}}=A_{p q} \neq 0$. Assim, $\left(\mathcal{H},\langle\cdot, \cdot\rangle_{\mathcal{H}}\right)$ tem a ortogonalidade a partir de suportes disjuntos se, e somente se, $A$ é diagonal.

\subsection{Ortogonalidade via suportes disjuntos e a função layout}

O objetivo principal desta seção é descrever uma condição necessaria e suficiente para que o EHR $\mathcal{H}_{K}$ possua a propriedade de ortogonalidade a partir de suportes disjuntos, via a função layout $\Phi$. Em todo o restante do capítulo, $K$ será um núcleo contínuo. Por questões de uniformização, escreveremos $\Phi(\emptyset)=\{0\}$.

Teorema 4.2.1. Sejam $K$ um núcleo PD sobre $E$ e $\Phi: E \rightarrow \mathcal{W}$ uma função layout para $K$. Suponha que a imagem de $\Phi$ seja um subconjunto fundamental de $\mathcal{W}$. Então, as seguintes afirmações são equivalentes:

(i) $\mathcal{H}_{K}$ possui ortogonalidade a partir de suportes disjuntos;

(ii) Se $U$ e $V$ são subconjuntos abertos de $E$ e $U \cup V=E$, então $\Phi(U)^{\perp} \perp \Phi(V)^{\perp}$. 
Demonstração: Suponhamos inicialmente que $(i i)$ vale e tomemos $f, g \in \mathcal{H}_{K}$ com suportes disjuntos. Pela Proposição 1.3.2, podemos escrever $f=\Phi_{u}$ e $g=\Phi_{v}$, com $u, v \in \mathcal{W}$. Ainda, a mesma proposição garante que

$$
\langle f, g\rangle_{\mathcal{H}_{K}}=\langle v, u\rangle_{\mathcal{W}}
$$

Escrevamos $A_{f}=E \backslash \operatorname{supp}(f)$ e $A_{g}=E \backslash \operatorname{supp}(g)$. Os conjuntos $A_{f}$ e $A_{g}$ são abertos em $E$ e

$$
E \backslash\left(A_{f} \cup A_{g}\right)=\left(E \backslash A_{f}\right) \cap\left(E \backslash A_{g}\right)=\operatorname{supp}(f) \cap \operatorname{supp}(g) .
$$

Como $f$ e $g$ possuem suportes disjuntos, concluímos que $A_{f} \cup A_{g}=E$. Por outro lado,

$$
0=f(x)=\langle\Phi(x), u\rangle_{\mathcal{W}}, \quad x \in A_{f}
$$

ou seja, $u \in \Phi\left(A_{f}\right)^{\perp}$. Analogamente, também temos que $v \in \Phi\left(A_{g}\right)^{\perp}$. Aplicando (ii) vemos que $\langle v, u\rangle_{\mathcal{W}}=0$ e, consequentemente, $\langle f, g\rangle_{\mathcal{H}_{K}}=0$. Isto demonstra que $\mathcal{H}_{K}$ possui ortogonalidade a partir de suportes disjuntos. Reciprocamente, suponha que $\mathcal{H}_{K}$ tenha tal propriedade e sejam $U$ e $V$ subconjuntos abertos de $E$ satisfazendo $E=U \cup V$. Para provar que $\Phi(U)^{\perp} \perp \Phi(V)^{\perp}$, sejam $u \in \Phi(U)^{\perp}$ e $v \in \Phi(V)^{\perp}$. É fácil ver que as aplicações $f:=\Phi_{u}$ e $g:=\Phi_{v}$ anulam-se em $U$ e $V$, respectivamente. Logo, $\operatorname{supp}\left(\Phi_{u}\right) \subseteq E \backslash U$ e $\operatorname{supp}\left(\Phi_{v}\right) \subseteq E \backslash V$. Em particular,

$$
\operatorname{supp}(f) \cap \operatorname{supp}(g) \subseteq(E \backslash U) \cap(E \backslash V)=E \backslash(U \cup V)=\emptyset .
$$

Pela condição $(i)$, concluímos que $\left\langle\Phi_{u}, \Phi_{v}\right\rangle_{\mathcal{H}_{K}}=0$. Retornando à primeira igualdade da demonstração, deduzimos que $\langle v, u\rangle_{\mathcal{W}}=0$. Assim, $(i)$ e (ii) são, de fato, afirmações equivalentes.

O corolário a seguir fornece uma roupagem alternativa para o teorema anterior.

Corolário 4.2.2. Nas condições do teorema anterior, as seguintes afirmações são equivalentes:

(i) $\mathcal{H}_{K}$ possui ortogonalidade a partir de suportes disjuntos;

(ii) Se $U$ e $V$ são subconjuntos abertos de $E$ e $U \cup V=E$, então $\Phi(U)^{\perp} \subseteq \overline{[\Phi(V)]}$ ou $\Phi(V)^{\perp} \subseteq \overline{[\Phi(U)]}$.

Demonstração: Se $\Phi(U)^{\perp} \perp \Phi(V)^{\perp}$, então

$$
\Phi(U)^{\perp} \subseteq \Phi(V)^{\perp \perp}=\overline{[\Phi(V)}^{\perp \perp}=\overline{[\Phi(V)]}
$$

e

$$
\Phi(V)^{\perp} \subseteq \Phi(U)^{\perp \perp}=\overline{[\Phi(U)}^{\perp \perp}=\overline{[\Phi(U)]} .
$$


Por outro lado, se uma das inclusões acima vale, digamos, a primeira delas, então

$$
\Phi(U)^{\perp} \subseteq \overline{[\Phi(V)]}=\Phi(V)^{\perp \perp}
$$

e, por conseguinte, $\Phi(U)^{\perp} \perp \Phi(V)^{\perp}$. Assim, a equivalência segue do Teorema 4.2.1.

A consequência a seguir é uma situação particular dos resultados anteriores.

Corolário 4.2.3. Sejam $K$ um núcleo PD sobre $E$ e $\Phi: E \rightarrow \mathcal{W}$ uma função layout para K. Se E é conexo e a imagem de $\Phi$ é um subconjunto fundamental de $\mathcal{W}$, então $\mathcal{H}_{K}$ possui a ortogonalidade a partir de suportes disjuntos.

Demonstração: Sejam $U$ e $V$ abertos de $E$ satisfazendo $U \cup V=E$. Sem perda de generalidade, suponhamos que tal união é disjunta (se não for, trocamos $V$ por $V \backslash U$ ). Neste caso, a união $U \cup V$ é uma cisão de $E$. Se $E$ é conexo, $E=U$ ou $E=V$. Desta forma, $\Phi(V)=\Phi(E)$ ou $\Phi(U)=\Phi(E)$. Se, além disso, $\Phi(E)$ é fundamental em $\mathcal{W}$, então

$$
\Phi(U)^{\perp}=\Phi(E)^{\perp}=\overline{[\Phi(E)}^{\perp}=\mathcal{W}^{\perp}=\{0\},
$$

ou $\Phi(V)^{\perp}=\{0\}$. Em particular, a fórmula $\Phi(U)^{\perp} \perp \Phi(V)^{\perp}$ é trivialmente satisfeita. Assim, o resultado é uma consequência do Teorema 4.2.1.

\subsection{Exemplificando}

Nesta última seção do trabalho, investigaremos a ortogonalidade a partir de suportes disjuntos no caso em que $\mathcal{H}_{K}$ tem dimensão finita. Para tanto, adotaremos as notações e conteúdos do Teorema 1.2.6 e do seu corolário. Vamos supor ainda que os suportes das aplicações $\phi_{j}$ são dois a dois disjuntos.

Teorema 4.3.1. Nas hipóteses do Teorema 1.2.6, suponha que as funções $\phi_{j}$ 's tenham suportes disjuntos. Então $\mathcal{H}_{K}$ possui ortogonalidade a partir de suportes disjuntos se, e somente se, A é uma matriz diagonal.

Demonstração: Inicialmente vamos supor que a matriz $A$ definida no Teorema 1.2.6 é diagonal. Uma vez que $A$ é positiva definida segue que $a_{i i}>0, i=1,2, \ldots, d$. Como nossa intenção é aplicar o Teorema 4.2.1, sejam $U$ e $V$ abertos de $E$ tais que $U \cup V=E$. Lembremos que a função layout para $K$ é dada por $\Phi(x)=\phi(x) A^{1 / 2}$, $x \in E$, onde $A^{1 / 2}=\left(b_{i j}\right)$ é a raíz quadrada de $A$ e $\mathcal{W}=\mathbb{C}^{d}$, munido de seu produto interno usual. Da hipótese de $A$ ser diagonal, segue trivialmente que $A^{1 / 2}$ também o é. Dando sequência, se $z=\left(z_{1}, z_{2}, \ldots, z_{d}\right) \in \Phi(U)^{\perp}$, temos que

$$
0=\langle z, \Phi(x)\rangle_{\mathbb{C}^{d}}=\left\langle z, \phi(x) A^{1 / 2}\right\rangle_{\mathbb{C}^{d}}=\sum_{j=1}^{d} b_{j j} z_{j} \overline{\phi_{j}(x)}, \quad x \in U .
$$


De maneira análoga, se $w=\left(w_{1}, w_{2}, \ldots, w_{d}\right) \in \Phi(V)^{\perp}$, então

$$
0=\langle w, \Phi(y)\rangle_{\mathbb{C}^{d}}=\sum_{j=1}^{d} b_{j j} w_{j} \overline{\phi_{j}(y)}, \quad y \in V .
$$

Como cada $\phi_{k}$ é não nula e estamos assumindo que os suportes das $\phi_{k}$ 's são disjuntos, tomamos pontos $x^{1}, x^{2}, \ldots, x^{d}$ em $E$ satisfazendo $\phi_{k}\left(x^{k}\right) \neq 0, k=1,2, \ldots, d$ e $\phi_{k}\left(x^{j}\right)=$ $0, j \neq k$. Como $E=U \cup V$ temos que considerar dois casos (para cada $i$ ): se $x^{i} \in U$, então a penúltima igualdade toma a forma

$$
0=b_{i i} z_{i} \overline{\phi_{i}\left(x^{i}\right)}
$$

e, portanto, $z_{i}=0$. Se $x^{i} \in V$, de maneira análoga obtemos que $w_{i}=0$. Essas argumentações implicam que $\langle z, w\rangle_{\mathbb{C}^{d}}=0$ e, portanto, $\Phi(U)^{\perp}$ e $\Phi(V)^{\perp}$ são ortogonais. Assim, o espaço $\mathcal{H}_{\Phi}$ possui a ortogonalidade a partir de suportes disjuntos. Sendo $\mathcal{H}_{K}$ uma cópia isométrica deste último, o mesmo vale para $\mathcal{H}_{K}$.

Reciprocamente, suponhamos que $A$ não seja uma matriz diagonal. Consequentemente, sua inversa $A^{-1}=\left(b_{i j}\right)$ também não é diagonal. Logo, existem índices $1 \leq r<s \leq d$ tais que $b_{r s} \neq 0$. Os conjuntos $U:=E \backslash \operatorname{supp}\left(\psi_{r}\right)$ e $V:=E \backslash \operatorname{supp}\left(\psi_{s}\right)$ são abertos em $E$ e, como os suportes das $\phi_{k}$ 's são disjuntos, temos que $U \cup V=E$. Ainda, $\operatorname{supp}\left(\phi_{j}\right) \subset U, j \neq r$ e $\operatorname{supp}\left(\phi_{i}\right) \subset V, i \neq s$. Para concluirmos os argumentos, é conveniente explicitarmos adequadamente ambos, $\Phi(U)^{\perp}$ e $\Phi(V)^{\perp}$. Observe que se $c \in \Phi(U)^{\perp}$, então

$$
0=\langle\Phi(x), c\rangle_{\mathbb{C}^{d}}=\left\langle\phi(x) A^{1 / 2}, c\right\rangle_{\mathbb{C}^{d}}, \quad x \in U .
$$

Expandindo o termo à direita obtemos

$$
\sum_{i=1}^{d} \phi_{i}(x)\left(A^{1 / 2} c^{*}\right)_{i}=0, \quad x \in U,
$$

onde $\left(A^{1 / 2} c^{*}\right)_{i}$ indica a $i$-ésima entrada do vetor $A^{1 / 2} c^{*}$. Como os suportes das $\phi_{k}$ 's são disjuntos, podemos tomar valores particulares de $x$ em supp $\left(\phi_{i}\right), i \neq r$, para deduzir da fórmula acima que $\left(A^{1 / 2} c^{*}\right)_{j}=0, j \neq r$. Em outras palavras,

$$
\Phi(U)^{\perp}=\left\{c \in \mathbb{C}^{d}:\left(A^{1 / 2} c^{*}\right)_{j}=0, \quad j \neq r\right\} .
$$

Analogamente,

$$
\Phi(V)^{\perp}=\left\{c \in \mathbb{C}^{d}:\left(A^{1 / 2} c^{*}\right)_{j}=0, \quad j \neq s\right\} .
$$

Agora é fácil notar que se $e_{j}$ indica o elemento canônico de $\mathbb{C}^{d}$ possuindo a $j$-ésima coordenada igual a 1 e zeros nas demais, então $\left(\left(A^{1 / 2}\right)^{-1} e_{r}{ }^{*}\right)^{*}$ e $\left(\left(A^{1 / 2}\right)^{-1} e_{s}{ }^{*}\right)^{*}$ são elementos de $\Phi(U)^{\perp}$ e $\Phi(V)^{\perp}$, respectivamente. Por outro lado,

$$
\left\langle\left(\left(A^{1 / 2}\right)^{-1} e_{s}^{*}\right)^{*},\left(\left(A^{1 / 2}\right)^{-1} e_{r}^{*}\right)^{*}\right\rangle_{\mathbb{C}^{d}}=b_{r s} \neq 0,
$$


ou seja, $\Phi(U)^{\perp}$ e $\Phi(V)^{\perp}$ não são ortogonais. 


\section{Referências Bibliográficas}

[1] Aronszajn, N., Theory of reproducing kernels. Trans. Amer. Math. Soc. 68, (1950). 337-404.

[2] Bachman, G.; Narici, L., Functional analysis. Academic Press, New York-London, 1966.

[3] Berg, C.; Christensen, J. P. R.; Ressel, P., Harmonic analysis on semigroups. Theory of positive definite and related functions. Graduate Texts in Mathematics, 100. Springer-Verlag, New York, 1984.

[4] Berlinet, A.; Thomas-Agnan, C., Reproducing kernel Hilbert spaces in probability and statistics. With a preface by Persi Diaconis. Kluwer Academic Publishers, Boston, MA, 2004.

[5] Berman, G.; Fryer, K. D., Introduction to combinatorics. Academic Press, New York-London, 1972.

[6] Bochner, S., Lectures on Fourier integrals. With an author's supplement on monotonic functions, Stieltjes integrals, and harmonic analysis. Translated by Morris Tenenbaum and Harry Pollard. Annals of Mathematics Studies, No. 42 Princeton University Press, Princeton, N.J., 1959

[7] Brezis, H., Functional analysis, Sobolev spaces and partial differential equations. Universitext. Springer, New York, 2011.

[8] Cheney, W.; Light, W., A course in approximation theory. Reprint of the 2000 original. Graduate Studies in Mathematics, 101. American Mathematical Society, Providence, RI, 2009. 
[9] Cucker, F.; Zhou, Ding-Xuan, Learning theory: an approximation theory viewpoint. With a foreword by Stephen Smale. Cambridge Monographs on Applied and Computational Mathematics. Cambridge University Press, Cambridge, 2007.

[10] Ferreira, J. C.; Menegatto, V. A., Eigenvalues of integral operators defined by smooth positive definite kernels. Integral Equations Operator Theory 64 (2009), no. $1,61-81$.

[11] Folland, G. B., Real analysis. Modern techniques and their applications. Second edition. Pure and Applied Mathematics (New York). A Wiley-Interscience Publication. John Wiley \& Sons, Inc., New York, 1999.

[12] Groemer, H., Geometric applications of Fourier series and spherical harmonics. (English summary) Encyclopedia of Mathematics and its Applications, 61. Cambridge University Press, Cambridge, 1996.

[13] Hewitt, E.; Stromberg, K., Real and abstract analysis. A modern treatment of the theory of functions of a real variable. Third printing. Graduate Texts in Mathematics, No. 25. Springer-Verlag New York-Heidelberg, 1975.

[14] Krantz, S. G., Function theory of several complex variables. Second edition. The Wadsworth \& Brooks/Cole Mathematics Series. Wadsworth \& Brooks/Cole Advanced Books \& Software, Pacific Grove, CA, 1992.

[15] Kreyszig, E., Introductory functional analysis with applications. Wiley Classics Library. John Wiley \& Sons, Inc., New York, 1989.

[16] Micchelli, C. A.; Xu, Yuesheng; Zhang, Haizhang, Universal kernels. J. Mach. Learn. Res. 7 (2006), 2651-2667.

[17] Oktumustur, B., Reproducing kernel Hilbert spaces, MSc. Thesis, Bilkent University, Turkey, 2005.

[18] Royden, L. H., Real Analysis. 3rd Edition, Macmillan Publishing Company, New York, 1988.

[19] Saitoh, Saburou, Theory of reproducing kernels and its applications. Pitman Research Notes in Mathematics Series, 189. Longman Scientific \& Technical, Harlow; copublished in the United States with John Wiley \& Sons, Inc., New York, 1988.

[20] Schoenberg, I. J., Positive definite functions on spheres. Duke Math. J. 9, (1942), 96-108. 
[21] Shawe-Taylor, J.; Cristianini, N., Kernel methods for pattern analysis. Cambridge University Press, 2004.

[22] Szegö, G., Orthogonal polynomials. Third edition. American Mathematical Society Colloquium Publications, Vol. 23. American Mathematical Society, Providence, R.I., 1967.

[23] Stone, M., The Generalized Weierstrass Approximation Theorem. Mathematics Magazine 21 \#5 (1948), 237-254.

[24] Stone, M, The Generalized Weierstrass Approximation Theorem. Mathematics Magazine 21 \#21 (1948), 167-184.

[25] Wendland, H., Scattered data approximation. Cambridge Monographs on Applied and Computational Mathematics, 17. Cambridge University Press, Cambridge, 2005.

[26] Young, N., An Introduction to Hilbert space. Cambridge University Press, 1988.

[27] Zhang, Haizhang, Orthogonality from disjoint support in reproducing kernel Hilbert spaces. J. Math. Anal. Appl. 349 (2009), no. 1, 201-210. 



\section{Índice Remissivo}

$G_{K}(X), 34$

$I_{\mu}, 30$

$K_{y}, 18$

$L, 30$

$\Phi_{X}(\mathcal{B}), 36$

$\Phi_{u}, 22$

$\iota, 30$

$\mathcal{M}(X), 30$

$j_{X}, 32$

$l_{X}, 35$

$w_{\Phi}^{\mu}, 31$

Anulador, 33

Base ortonormal para um espaço de Hilbert, 36

Complemento ortogonal, 18

Completamento de espaço vetorial, 17

Conjunto

de unicidade, 50

fundamental, 23

total, 23

Espaço

de Hilbert de reprodução, 18

layout, 17

Função layout, 17

Função layout universal, 37
Medida

absolutamente contínua, 50

de Radon, 30

de variação total, 30

Medidas mutualmente singulares, 51

Núcleo

de Mercer, 14, 43

invariante por translação, 47

positivo definido, 15

radial, 52

universal, 29, 37

Operador integral, 38

Ortogonalidade a partir de suportes disjuntos, 57

Polinômios

de Legendre, 16, 46

ultra-esférico, 16

Propriedade universal da aproximação, 37

Suporte

de uma função, 51, 57

de uma medida, 48

Teorema

de Decomposição de Lebesgue, 50

de Fubini-Tonelli, 32 
de Radon-Nikodym, 51

de Representação de Riesz, 30, 31

multinomial, 45, 53

de Hahn-Banach, 34

de Stone-Weierstrass, 45

Teoria de Mercer, 20, 38, 43

Variação total, 30 